\title{
Multiobjective Fractional Programming Problems and Second Order Generalized Hybrid Invexity Frameworks
}

\author{
Ram U. Verma ${ }^{1, *}$ \\ ${ }^{1}$ Department of Mathematics, Texas State University, USA
}

Received: 15 July 2014; Accepted: 7 October 2014

Editor: Chen Ling

\begin{abstract}
In this paper, the parametrically generalized sufficient efficiency conditions for multiobjective fractional programming based on the hybrid $(\Phi, \rho, \eta, \zeta, \theta)$-invexities are developed, and then efficient solutions to the multiobjective fractional programming problems are established. Furthermore, the obtained results on sufficient efficiency conditions are generalized to the case of the $\epsilon$-efficient solutions. The results thus obtained generalize and unify a wider range of investigations on the theory and applications to the multiobjective fractional programming based on the hybrid $(\Phi, \rho, \eta, \zeta, \theta)$-invexity frameworks.
\end{abstract}

Keywords Generalized invexity, Multiobjective fractional programming, Efficient solutions, $\epsilon$-efficient solutions, Parametric sufficient efficiency conditions

\section{AMS 2010 subject classifications 90C $32,90 \mathrm{C} 45$}

DOI: $10.19139 /$ soic.v2i4.92

\section{Introduction}

Mishra and Rueda [11] introduced higher order generalized invexity and duality models in mathematical programming, while Mangasarian [8] focused on the second order duality for a conventional nonlinear programming problem, where the approach is based on constructing a second order dual problem by taking linear and quadratic approximations of the objective and constraint functions for an arbitrary but fixed point leading to the Wolfe dual model for the approximated

\footnotetext{
*Correspondence to: Department of Mathematics,Texas State University, San Marcos, TX 78666, USA. Email:verma99@msn.com
}

ISSN 2310-5070 (online) ISSN 2311-004X (print)

Copyright (C) 2014 International Academic Press 
problem, while letting the fixed point to vary. Verma [24] investigated the second order $(\rho, \eta, \theta)$-invexities to the context of parametric sufficient optimality conditions in semiinfinite discrete minimax fractional programming. Zalmai and Zhang [38] have established a set of necessary efficiency conditions and a fairly large number of global nonparametric sufficient efficiency results under various frameworks for generalized $(\eta, \rho)$-invexity for the semiinfinite discrete minimax fractional programming. Just recently, Verma [22] investigated a general framework for a class of $(\rho, \eta, \theta)$-invex functions to examine some parametric sufficient efficiency conditions for multiobjective fractional programming problems for weakly $\epsilon$-efficient solutions. Inspired by these research developments, we first introduce the hybrid $(\Phi, \rho, \eta, \zeta, \theta)$-invexities as well as the second order hybrid $(\Phi, \rho, \eta, \zeta, \theta)$-invexities, second, introduce some parametrically sufficient efficiency conditions for multiobjective fractional programming, and finally, explore the efficient solutions to multiobjective fractional programming problems. The results established in this communication, not only generalize (and unify) the results on general sufficient efficiency conditions for multiobjective fractional programming problems based on the hybrid invexity of functions, but also generalize second order invexity results to more general settings. We consider, based on the generalized hybrid $(\Phi, \rho, \eta, \zeta, \theta)$-invexities of functions, the following multiobjective fractional programming problem: $(\mathrm{P})$

$$
\text { Minimize }\left(\frac{f_{1}(x)}{g_{1}(x)}, \frac{f_{2}(x)}{g_{2}(x)}, \cdots, \frac{f_{p}(x)}{g_{p}(x)}\right)
$$

subject to $x \in Q=\left\{x \in X: H_{j}(x) \leq 0, j \in\{1,2, \cdots, m\}\right\}$, where $X$ is an open convex subset of $\Re^{n}$ (n-dimensional Euclidean space), $f_{i}$ and $g_{i}$ for $i \in\{1, \cdots, p\}$, and $H_{j}$ for $j \in\{1, \cdots, m\}$, are real-valued functions defined on $X$ such that $f_{i}(x) \geq 0, g_{i}(x)>0$ for $i \in\{1, \cdots, p\}$ and for all $x \in Q$. Here $Q$ denotes the feasible set of $(\mathrm{P})$.

Next, we observe that problem $(\mathrm{P})$ is equivalent to the nonfractional programming problem: $(\mathrm{P} \lambda)$

$$
\operatorname{Minimize}\left(f_{1}(x)-\lambda_{1} g_{1}(x), \cdots, f_{p}(x)-\lambda_{p} g_{p}(x)\right)
$$

subject to $x \in Q$ with

$$
\lambda=\left(\lambda_{1}, \lambda_{2}, \cdots, \lambda_{p}\right)=\left(\frac{f_{1}\left(x^{*}\right)}{g_{1}\left(x^{*}\right)}, \frac{f_{2}\left(x^{*}\right)}{g_{2}\left(x^{*}\right)}, \cdots, \frac{f_{p}\left(x^{*}\right)}{g_{p}\left(x^{*}\right)}\right),
$$

where $x^{*}$ is an efficient solution to $(\mathrm{P})$.

We observe that general mathematical programming problems offer a great opportunity for applications to other fields, for instance, applications to game theory, statistical analysis, engineering design (including design of 
control systems, design of earthquakes-resistant structures, digital filters, and electronic circuits), random graphs, boundary value problems, wavelet analysis, environmental protection planning, decision and management sciences, optimal control problems, continuum mechanics, robotics, and others. For more details on generalized efficiency and efficiency results and applications, we recommend the reader [1]-[41].

This submission is organized as follows: the introductory section deals with a brief historical development for the multiobjective fractional mathematical programming, while emphasizing the roles of the generalized invex functions. In Section 2, the hybrid $(\Phi, \rho, \eta, \zeta, \theta)$-invex functions of higher orders are introduced, and Section 3 deals with sufficient efficiency conditions leading to the solvability of the problem $(\mathrm{P})$ using the hybrid $(\Phi, \rho, \eta, \zeta, \theta)$-invexities.

\section{Hybrid Invexities}

In this section, we introduce and develop some concepts and notations for the problem on hand based on the $(\alpha, \eta)$-V- invexities introduced by Mond and Zhang [15], and recently generalized by Zalmai [35, 36, 37]. Let $X$ be an open convex subset of $\Re^{n}$ (n-dimensional Euclidean space). Let $\langle\cdot, \cdot\rangle$ denote the inner product, and let $z \in \Re^{n}$. Suppose that $f: X \rightarrow \Re$ is a real-valued twice continuously differentiable function defined on $X$, and that $\nabla f(y)$ and $\nabla^{2} f(y)$ denote, respectively, the gradient and Hessian of $f$ at $y$.

Definition 2.1. A twice differentiable function $f: X \rightarrow \Re$ is said to be hybrid $(\Phi, \rho, \eta, \zeta, \theta)$-invex at $x^{*}$ of second order if there exists a function $\Phi: \Re \rightarrow \Re$ such that for each $x \in X, \rho: X \times X \rightarrow \Re, \eta, \zeta, \theta: X \times X \rightarrow \Re^{n}$, and $z \in \Re^{n}$,

$$
\begin{aligned}
\Phi\left(f(x)-f\left(x^{*}\right)\right) \geq & \left\langle\nabla f\left(x^{*}\right), \eta\left(x, x^{*}\right)\right\rangle+\left\langle\nabla^{2} f\left(x^{*}\right) z, \zeta\left(x, x^{*}\right)\right\rangle \\
& -\frac{1}{2}\left\langle\nabla^{2} f\left(x^{*}\right) z, z\right\rangle+\rho\left(x, x^{*}\right)\left\|\theta\left(x, x^{*}\right)\right\|^{2} .
\end{aligned}
$$

Definition 2.2. A twice differentiable function $f: X \rightarrow \Re$ is said to be hybrid $(\Phi, \rho, \eta, \zeta, \theta)$-pseudo-invex at $x^{*}$ of second order if there exists a function $\Phi$ : $\Re \rightarrow \Re$ such that for each $x \in X, \rho: X \times X \rightarrow \Re, \eta, \zeta, \theta: X \times X \rightarrow \Re^{n}$, and $z \in \Re^{n}$,

$$
\begin{aligned}
& \left\langle\nabla f\left(x^{*}\right), \eta\left(x, x^{*}\right)\right\rangle+\left\langle\nabla^{2} f\left(x^{*}\right) z, \zeta\left(x, x^{*}\right)\right\rangle-\frac{1}{2}\left\langle\nabla^{2} f\left(x^{*}\right) z, z\right\rangle \\
& +\rho\left(x, x^{*}\right)\left\|\theta\left(x, x^{*}\right)\right\|^{2} \geq 0 \\
& \Rightarrow \Phi\left(f(x)-f\left(x^{*}\right)\right) \geq 0 .
\end{aligned}
$$

Definition 2.3. A twice differentiable function $f: X \rightarrow \Re$ is said to be strictly hybrid $(\Phi, \rho, \eta, \zeta, \theta)$ - pseudo-invex at $x^{*}$ of second order if there exists a function 
$\Phi: \Re \rightarrow \Re$ such that for each $x \in X, \rho: X \times X \rightarrow \Re, \eta, \zeta, \theta: X \times X \rightarrow \Re^{n}$, and $z \in \Re^{n}$,

$$
\begin{aligned}
& \left\langle\nabla f\left(x^{*}\right), \eta\left(x, x^{*}\right)\right\rangle+\left\langle\nabla^{2} f\left(x^{*}\right) z, \zeta\left(x, x^{*}\right)\right\rangle-\frac{1}{2}\left\langle\nabla^{2} f\left(x^{*}\right) z, z\right\rangle \\
& +\rho\left(x, x^{*}\right)\left\|\theta\left(x, x^{*}\right)\right\|^{2} \geq 0 \\
& \Rightarrow \Phi\left(f(x)-f\left(x^{*}\right)\right)>0 .
\end{aligned}
$$

Definition 2.4. A twice differentiable function $f: X \rightarrow \Re$ is said to be prestrictly hybrid $(\Phi, \rho, \eta, \zeta, \theta)$-pseudo-invex at $x^{*}$ of second order if there exists a function $\Phi: \Re \rightarrow \Re$ such that for each $x \in X, \rho: X \times X \rightarrow \Re, \eta, \zeta, \theta: X \times X \rightarrow \Re^{n}$, and $z \in \Re^{n}$,

$$
\begin{aligned}
& \left\langle\nabla f\left(x^{*}\right), \eta\left(x, x^{*}\right)\right\rangle+\left\langle\nabla^{2} f\left(x^{*}\right) z, \zeta\left(x, x^{*}\right)\right\rangle-\frac{1}{2}\left\langle\nabla^{2} f\left(x^{*}\right) z, z\right\rangle \\
& +\rho\left(x, x^{*}\right)\left\|\theta\left(x, x^{*}\right)\right\|^{2}>0 \\
& \Rightarrow \Phi\left(f(x)-f\left(x^{*}\right)\right) \geq 0 .
\end{aligned}
$$

Definition 2.5. A twice differentiable function $f: X \rightarrow \Re$ is said to be hybrid $(\Phi, \rho, \eta, \zeta, \theta)$-quasi-invex at $x^{*}$ of second order if there exists a function $\Phi: \Re \rightarrow$ $\Re$ such that for each $x \in X, \rho: X \times X \rightarrow \Re, \eta, \zeta, \theta: X \times X \rightarrow \Re^{n}$, and $z \in \Re^{n}$,

$$
\begin{aligned}
& \Phi\left(f(x)-f\left(x^{*}\right)\right) \leq 0 \\
& \Rightarrow\left\langle\nabla f\left(x^{*}\right), \eta\left(x, x^{*}\right)\right\rangle+\left\langle\nabla^{2} f\left(x^{*}\right) z, \zeta\left(x, x^{*}\right)\right\rangle-\frac{1}{2}\left\langle\nabla^{2} f\left(x^{*}\right) z, z\right\rangle \\
& +\rho\left(x, x^{*}\right)\left\|\theta\left(x, x^{*}\right)\right\|^{2} \leq 0 .
\end{aligned}
$$

Definition 2.6. A twice differentiable function $f: X \rightarrow \Re$ is said to be strictly hybrid $(\Phi, \rho, \eta, \zeta, \theta)$ - quasi-invex at $x^{*}$ of second order if there exists a function $\Phi: \Re \rightarrow \Re$ such that for each $x \in X, \rho: X \times X \rightarrow \Re, \eta, \zeta, \theta: X \times X \rightarrow \Re^{n}$, and $z \in \Re^{n}$,

$$
\begin{aligned}
& \Phi\left(f(x)-f\left(x^{*}\right)\right) \leq 0 \\
& \Rightarrow\left\langle\nabla f\left(x^{*}\right), \eta\left(x, x^{*}\right)\right\rangle+\left\langle\nabla^{2} f\left(x^{*}\right) z, \zeta\left(x, x^{*}\right)\right\rangle-\frac{1}{2}\left\langle\nabla^{2} f\left(x^{*}\right) z, z\right\rangle \\
& +\rho\left(x, x^{*}\right)\left\|\theta\left(x, x^{*}\right)\right\|^{2}<0 .
\end{aligned}
$$

Definition 2.7. A twice differentiable function $f: X \rightarrow \Re$ is said to be prestrictly hybrid $(\Phi, \rho, \eta, \zeta, \theta)$ - quasi-invex at $x^{*}$ of second order if there exists a function 
$\Phi: \Re \rightarrow \Re$ such that for each $x \in X, \rho: X \times X \rightarrow \Re, \eta, \zeta, \theta: X \times X \rightarrow \Re^{n}$, and $z \in \Re^{n}$,

$$
\begin{aligned}
& \Phi\left(f(x)-f\left(x^{*}\right)\right)<0 \\
\Rightarrow \quad & \left\langle\nabla f\left(x^{*}\right), \eta\left(x, x^{*}\right)\right\rangle+\left\langle\nabla^{2} f\left(x^{*}\right) z, \zeta\left(x, x^{*}\right)\right\rangle-\frac{1}{2}\left\langle\nabla^{2} f\left(x^{*}\right) z, z\right\rangle \\
& +\rho\left(x, x^{*}\right)\left\|\theta\left(x, x^{*}\right)\right\|^{2} \leq 0,
\end{aligned}
$$

equivalently,

$$
\begin{aligned}
& \left\langle\nabla f\left(x^{*}\right), \eta\left(x, x^{*}\right)\right\rangle+\left\langle\nabla^{2} f\left(x^{*}\right) z, \zeta\left(x, x^{*}\right)\right\rangle-\frac{1}{2}\left\langle\nabla^{2} f\left(x^{*}\right) z, z\right\rangle \\
& +\rho\left(x, x^{*}\right)\left\|\theta\left(x, x^{*}\right)\right\|^{2}>0 \\
\Rightarrow \quad & \Phi\left(f(x)-f\left(x^{*}\right)\right) \geq 0 .
\end{aligned}
$$

\section{Some Examples and Remarks}

We observe that Definition 2.1 reduces to Mond and Zhang [15], and Zalmai [37] for $\zeta\left(x, x^{*}\right)=\eta\left(x, x^{*}\right)$, while similar examples hold for Definitions 2.2 - 2.7.

Example 2.1. The function $f$ is said to be second-order $(\Phi, \rho, \eta, \theta)$-V-invex at $x^{*}$ if there exist functions $\Phi: \Re \rightarrow \Re, \rho: X \times X \rightarrow \Re$ and $\eta, \theta: X \times X \rightarrow \Re^{n}$ such that for each $x \in X, z \in \Re^{n}$,

$$
\begin{gathered}
\Phi\left(f(x)-f\left(x^{*}\right)\right) \geqq\left\langle\left[\nabla f\left(x^{*}\right)+\nabla^{2} f\left(x^{*}\right) z\right], \eta\left(x, x^{*}\right)\right\rangle-\left\langle\frac{1}{2} \nabla^{2} f\left(x^{*}\right) z, z\right\rangle \\
+\rho\left(x, x^{*}\right)\left\|\theta\left(x, x^{*}\right)\right\|^{2} .
\end{gathered}
$$

When function $f$ is first-order differentiable, Definition 2.1 unifies most of the well-explored notions of the invexities in the literature.

Example 2.2. A differentiable function $f: X \rightarrow \Re$ is said to be $(\Phi, \rho, \eta, \theta)$-invex at $x^{*}$ of first order if there exists a function $\Phi: \Re \rightarrow \Re$ such that for each $x \in X$, $\rho: X \times X \rightarrow \Re$, and $\eta, \theta: X \times X \rightarrow \Re^{n}$,

$$
\Phi\left(f(x)-f\left(x^{*}\right)\right) \geq\left\langle\nabla f\left(x^{*}\right), \eta\left(x, x^{*}\right)\right\rangle+\rho\left(x, x^{*}\right)\left\|\theta\left(x, x^{*}\right)\right\|^{2} .
$$

Definition 2.8. A point $x^{*} \in Q$ is an efficient solution to (P) if there exists no $x \in Q$ such that

$$
\frac{f_{i}(x)}{g_{i}(x)} \leq \frac{f_{i}\left(x^{*}\right)}{g_{i}\left(x^{*}\right)} \forall i=1, \cdots, p,
$$

Stat., Optim. Inf. Comput. Vol. 2, December 2014. 


$$
\frac{f_{j}(x)}{g_{j}(x)}<\frac{f_{j}\left(x^{*}\right)}{g_{j}\left(x^{*}\right)} \text { for some } j \in\{1, \cdots, p\} .
$$

Next to this context, we have the following auxiliary problem:

$$
\operatorname{Minimize}_{x \in Q}\left(f_{1}(x)-\bar{\lambda}_{1} g_{1}(x), \cdots, f_{p}(x)-\bar{\lambda}_{p} g_{p}(x)\right),
$$

subject to $x \in Q$,

where $\bar{\lambda}_{i}$ for $i \in\{1, \cdots, p\}$ are parameters, and $\bar{\lambda}_{i}=\frac{f_{i}\left(x^{*}\right)}{g_{i}\left(x^{*}\right)}$.

Next, we introduce the efficiency solvability conditions for $(\mathrm{P} \bar{\lambda})$ problem.

Definition 2.9. A point $x^{*} \in Q$ is an efficient solution to $(\mathrm{P} \bar{\lambda})$ if there does not exist an $x \in Q$ such that

$$
\begin{gathered}
f_{i}(x)-\bar{\lambda}_{i} g_{i}(x) \leq f_{i}\left(x^{*}\right)-\bar{\lambda}_{i} g_{i}\left(x^{*}\right) \forall i=1, \cdots, p, \\
f_{j}(x)-\bar{\lambda}_{j} g_{j}(x)<f_{j}\left(x^{*}\right)-\bar{\lambda}_{j} g_{j}\left(x^{*}\right) \text { for some } j \in\{1, \cdots, p\},
\end{gathered}
$$

where $\bar{\lambda}_{i}=\frac{f_{i}\left(x^{*}\right)}{g_{i}\left(x^{*}\right)}$ for $i=1, \cdots, p$.

Next, we recall the following result (Verma [24]) that provides a set of necessary efficiency conditions for problem $(P)$ to developing some sufficient efficiency conditions for the next section based on second order $(\Phi, \rho, \eta, \zeta, \theta)$-invexities.

Theorem 2.1. [24] Let $x^{*} \in \mathbb{F}$ and $\lambda^{*}=\max _{1 \leq i \leq p} f_{i}\left(x^{*}\right) / g_{i}\left(x^{*}\right)$ for each $i \in \underline{p}$, and let $f_{i}$ and $g_{i}$ be twice continuously differentiable at $x^{*}$ for each $i \in \underline{p}$. For each $j \in \underline{q}$, let the function $z \rightarrow G_{j}(z, t)$ be twice continuously differentiable at $x^{*}$ for all $t \in T_{j}$, and for each $k \in \underline{r}$, let the function $z \rightarrow H_{k}(z, s)$ be twice continuously differentiable at $x^{*}$ for all $s \in S_{k}$. If $x^{*}$ is an efficient solution of (P), if the second order generalized Abadie constraint qualification holds at $x^{*}$, and if for any critical direction $y$, the set cone

$$
\begin{aligned}
& \left\{\left(\nabla G_{j}\left(x^{*}, t\right),\left\langle y, \nabla^{2} G_{j}\left(x^{*}, t\right) y\right\rangle\right): t \in \hat{T}_{j}\left(x^{*}\right), j \in \underline{q}\right\} \\
+ & \operatorname{span}\left\{\left(\nabla H_{k}\left(x^{*}, s\right),\left\langle y, \nabla^{2} H_{k}\left(x^{*}, s\right) y\right\rangle\right): s \in S_{k}, k \in \underline{r}\right\}, \\
& \text { where } \hat{T}_{j}\left(x^{*}\right) \equiv\left\{t \in T_{j}: G_{j}\left(x^{*}, t\right)=0\right\},
\end{aligned}
$$

is closed, then there exist $u^{*} \in U \equiv\left\{u \in \mathbb{R}^{p}: u \geq 0, \sum_{i=1}^{p} u_{i}=1\right\}$ and integers $\nu_{0}^{*}$ and $\nu^{*}$ with $0 \leq \nu_{0}^{*} \leq \nu^{*} \leq n+1$ such that there exist $\nu_{0}^{*}$ indices $j_{m}$ with $1 \leq j_{m} \leq q$ together with $\nu_{0}^{*}$ points $t^{m} \in \hat{T}_{j_{m}}\left(x^{*}\right), m \in \nu_{0}^{*}, \nu^{*}-\nu_{0}^{*}$ indices $k_{m}$ with $1 \leq k_{m} \leq r$ together with $\nu^{*}-\nu_{0}^{*}$ points $s^{m} \in S_{k_{m}}$ for $m \in \underline{\nu^{*}} \backslash \underline{\nu_{0}^{*}}$, and $\nu^{*}$ 
real numbers $v_{m}^{*}$ with $v_{m}^{*}>0$ for $m \in \underline{\nu_{0}^{*}}$ with the property that

$$
\begin{aligned}
& \sum_{i=1}^{p} u_{i}^{*}\left[\nabla f_{i}\left(x^{*}\right)-\lambda^{*}\left(\nabla g_{i}\left(x^{*}\right)\right]+\sum_{m=1}^{\nu_{0}^{*}} v_{m}^{*}\left[\nabla G_{j_{m}}\left(x^{*}, t^{m}\right)\right.\right. \\
& +\sum_{m=\nu_{0}^{*}+1}^{\nu^{*}} v_{m}^{*} \nabla H_{k}\left(x^{*}, s^{m}\right)=0, \\
& \left\langle y,\left[\sum_{i=1}^{p} u_{i}^{*}\left[\nabla^{2} f_{i}\left(x^{*}\right)-\lambda^{*} \nabla^{2} g_{i}\left(x^{*}\right)\right]+\sum_{m=1}^{\nu_{0}^{*}} v_{m}^{*} \nabla^{2} G_{j_{m}}\left(x^{*}, t^{m}\right)\right.\right. \\
& \left.\left.+\sum_{m=\nu_{0}^{*}+1}^{\nu^{*}} v_{m}^{*} \nabla^{2} H_{k}\left(x^{*}, s^{m}\right)\right] y\right\rangle \geq 0, \\
& u_{i}^{*}\left[f_{i}\left(x^{*}\right)-\lambda^{*} g_{i}\left(x^{*}\right)\right]=0, \quad i \in \underline{p},
\end{aligned}
$$

where $\underline{\nu} \backslash \underline{\nu_{0}}$ is the complement of the set $\underline{\nu_{0}}$ relative to the set $\underline{\nu}$.

\section{Efficiency Conditions for Problem (P)}

This section deals with some parametrically sufficient efficiency conditions for problem $(\mathrm{P})$ under the hybrid frameworks for $(\Phi, \rho, \eta, \zeta, \theta)$-invexities. We begin with real-valued functions $E_{i}\left(., x^{*}, u^{*}\right)$ and $B_{j}(., v)$ defined by

$$
E_{i}\left(x, x^{*}, u^{*}\right)=u_{i}\left[f_{i}(x)-\left(\frac{f_{i}\left(x^{*}\right)}{g_{i}\left(x^{*}\right)}\right) g_{i}(x)\right], i \in\{1, \cdots, p\}
$$

and

$$
B_{j}(., v)=v_{j} H_{j}(x), j=1, \cdots, m
$$

Theorem 3.1. Let $x^{*} \in Q$, let $f_{i}, g_{i}$ for $i \in\{1, \cdots, p\}$ with $\frac{f_{i}\left(x^{*}\right)}{g_{i}\left(x^{*}\right)} \geq 0, g_{i}\left(x^{*}\right)>0$ and $H_{j}$ for $j \in\{1, \cdots, m\}$ be twice continuously differentiable at $x^{*} \in Q$, and let there exist $u^{*} \in U=\left\{u \in \Re^{p}: u>0, \Sigma_{i=1}^{p} u_{i}=1\right\}$ and $v^{*} \in \Re_{+}^{m}$ such that

$$
\sum_{i=1}^{p} u_{i}^{*}\left[\nabla f_{i}\left(x^{*}\right)-\left(\frac{f_{i}\left(x^{*}\right)}{g_{i}\left(x^{*}\right)}\right) \nabla g_{i}\left(x^{*}\right)\right]+\sum_{j=1}^{m} v_{j}^{*} \nabla H_{j}\left(x^{*}\right)=0,
$$




$$
\begin{aligned}
& \left\langle\left[\sum_{i=1}^{p} u_{i}^{*}\left[\nabla^{2} f_{i}\left(x^{*}\right)-\left(\frac{f_{i}\left(x^{*}\right)}{g_{i}\left(x^{*}\right)}\right) \nabla^{2} g_{i}\left(x^{*}\right)\right]+\sum_{j=1}^{m} v_{j}^{*} \nabla^{2} H_{j}\left(x^{*}\right)\right] z, \zeta\left(x, x^{*}\right)\right\rangle \\
& -\frac{1}{2}\left\langle\left[\sum_{i=1}^{p} u_{i}^{*}\left[\nabla^{2} f_{i}\left(x^{*}\right)-\left(\frac{f_{i}\left(x^{*}\right)}{g_{i}\left(x^{*}\right)}\right) \nabla^{2} g_{i}\left(x^{*}\right)\right]\right.\right. \\
& \left.\left.+\sum_{j=1}^{m} v_{j}^{*} \nabla^{2} H_{j}\left(x^{*}\right)\right] z, z\right\rangle \geq 0
\end{aligned}
$$

and

$$
v_{j}^{*} H_{j}\left(x^{*}\right)=0, j \in\{1, \cdots, m\} .
$$

Suppose, in addition, that any one of the following assumptions holds (for $\left.\rho\left(x, x^{*}\right) \geq 0\right)$ :

(i) $E_{i}\left(. ; x^{*}, u^{*}\right) \quad \forall i \in\{1, \cdots, p\} \quad$ are hybrid $(\Phi, \rho, \eta, \zeta, \theta)$-pseudo-invex at $x^{*}$ with $\bar{\Phi}(a) \geq 0 \Rightarrow a \geq 0$, and $B_{j}\left(., v^{*}\right) \quad \forall j \in\{1, \cdots, m\}$ are $\operatorname{hybrid}(\tilde{\Phi}, \bar{\rho}, \eta, \zeta, \theta)$-quasi-invex at $x^{*}$ for $\tilde{\Phi}$ increasing and $\tilde{\Phi}(0)=0$.

(ii) $E_{i}\left(. ; x^{*}, u^{*}\right) \forall i \in\{1, \cdots, p\}$ are prestrictly hybrid $(\Phi, \rho, \eta, \zeta, \theta)$-pseudoinvex at $x^{*}$ for $\Phi(a) \geq 0 \Rightarrow a \geq 0$, and $B_{j}\left(., v^{*}\right) \forall j \in\{1, \cdots, m\}$ are strictly hybrid $(\tilde{\Phi}, \bar{\rho}, \eta, \zeta, \theta)$-quasi-invex at $x^{*}$ for $\tilde{\Phi}$ increasing and $\tilde{\Phi}(0)=0$.

(iii) $E_{i}\left(. ; x^{*}, u^{*}\right) \forall i \in\{1, \cdots, p\}$ are prestrictly hybrid $(\Phi, \rho, \eta, \zeta, \theta)$-quasiinvex at $x^{*}$ for $\Phi(a) \geq 0 \Rightarrow a \geq 0$, and $B_{j}\left(., v^{*}\right) \forall j \in\{1, \cdots, m\}$ are strictly hybrid $(\tilde{\Phi}, \bar{\rho}, \eta, \zeta, \theta)$-quasi-invex at $x^{*}$ for $\tilde{\Phi}$ increasing and $\tilde{\Phi}(0)=0$.

(iv) For each $i \in\{1, \cdots, p\}, f_{i}$ is hybrid $\left(\Phi, \rho_{1}, \eta, \zeta, \theta\right)$-invex and $-g_{i}$ is hybrid $\left(\Phi, \rho_{2}, \eta, \zeta, \theta\right)$-invex at $x^{*}$ for $\Phi(a) \geq 0 \Rightarrow a \geq 0$, and $H_{j}\left(., v^{*}\right) \forall j \in$ $\{1, \cdots, m\}$ is hybrid $\left(\bar{\Phi}, \rho_{3}, \eta, \zeta, \theta\right)$-quasi-invex at $x^{*}$ for $\bar{\Phi}$ increasing and $\bar{\Phi}(0)=0$, and $\Sigma_{j=1}^{m} v_{j}^{*} \rho_{3}\left(x, x^{*}\right)+\rho^{*} \geq 0$ for $\rho^{*}=\Sigma_{i=1}^{p} u_{i}^{*}\left(\rho_{1}\left(x, x^{*}\right)+\right.$ $\left.\phi\left(x^{*}\right) \rho_{2}\left(x, x^{*}\right)\right)$ and for $\phi\left(x^{*}\right)=\frac{f_{i}\left(x^{*}\right)}{g_{i}\left(x^{*}\right)}$.

Then $x^{*}$ is an efficient solution to $(\mathrm{P})$.

Stat., Optim. Inf. Comput. Vol. 2, December 2014. 
Proof

If (i) holds, and if $x \in Q$, then it follows from (3.1) and (3.2) that

$$
\begin{aligned}
& \left\langle\sum_{i=1}^{p} u_{i}^{*}\left[\nabla f_{i}\left(x^{*}\right)-\left(\frac{f_{i}\left(x^{*}\right)}{g_{i}\left(x^{*}\right)}\right) \nabla g_{i}\left(x^{*}\right)\right], \eta\left(x, x^{*}\right)\right\rangle \\
& +\left\langle\sum_{i=1}^{p} u_{i}^{*}\left[\nabla^{2} f_{i}\left(x^{*}\right) z-\left(\frac{f_{i}\left(x^{*}\right)}{g_{i}\left(x^{*}\right)}\right) \nabla^{2} g_{i}\left(x^{*}\right) z\right], \zeta\left(x, x^{*}\right)\right\rangle \\
& -\frac{1}{2}\left\langle\sum_{i=1}^{p} u_{i}^{*}\left[\nabla^{2} f_{i}\left(x^{*}\right) z-\left(\frac{f_{i}\left(x^{*}\right)}{g_{i}\left(x^{*}\right)}\right) \nabla^{2} g_{i}\left(x^{*}\right) z\right], z\right\rangle \\
& +\left\langle\sum_{j=1}^{m} v_{j}^{*} \nabla H_{j}\left(x^{*}\right), \eta\left(x, x^{*}\right)\right\rangle+\left\langle\Sigma_{j=1}^{m} v_{j}^{*} \nabla^{2} H_{j}\left(x^{*}\right) z, \zeta\left(x, x^{*}\right)\right\rangle \\
& -\frac{1}{2}\left\langle\sum_{j=1}^{m} v_{j}^{*} \nabla^{2} H_{j}\left(x^{*}\right) z, z\right\rangle \geq 0 .
\end{aligned}
$$

Since $v^{*} \geq 0, x \in Q$ and (3.3) holds, we have

$$
\sum_{j=1}^{m} v_{j}^{*} H_{j}(x) \leq 0=\sum_{j=1}^{m} v_{j}^{*} H_{j}\left(x^{*}\right),
$$

and in light of assumptions on $\tilde{\Phi}$, we find

$$
\tilde{\Phi}\left(\Sigma_{j=1}^{m} v_{j}^{*} H_{j}(x)-\Sigma_{j=1}^{m} v_{j}^{*} H_{j}\left(x^{*}\right)\right) \leq 0,
$$

which applying the hybrid $(\tilde{\Phi}, \bar{\rho}, \eta, \zeta, \theta)$-quasi-invexity of $B_{j}\left(., v^{*}\right)$ at $x^{*}$, results in

$$
\begin{aligned}
& \left\langle\sum_{j=1}^{m} v_{j}^{*} \nabla H_{j}\left(x^{*}\right), \eta\left(x, x^{*}\right)\right\rangle+\left\langle\sum_{j=1}^{m} v_{j}^{*} \nabla^{2} H_{j}\left(x^{*}\right) z, \zeta\left(x, x^{*}\right)\right\rangle \\
& -\frac{1}{2}\left\langle\Sigma_{j=1}^{m} v_{j}^{*} \nabla^{2} H_{j}\left(x^{*}\right) z, z\right\rangle+\bar{\rho}\left(x, x^{*}\right)\left\|\theta\left(x, x^{*}\right)\right\|^{2} \leq 0 .
\end{aligned}
$$

It follows from (3.4) and (3.5) that

$$
\begin{aligned}
& \left\langle\sum_{i=1}^{p} u_{i}^{*}\left[\nabla f_{i}\left(x^{*}\right)-\left(\frac{f_{i}\left(x^{*}\right)}{g_{i}\left(x^{*}\right)}\right) \nabla g_{i}\left(x^{*}\right)\right], \eta\left(x, x^{*}\right)\right\rangle \\
+ & \left\langle\sum_{i=1}^{p} u_{i}^{*}\left[\nabla^{2} f_{i}\left(x^{*}\right) z-\left(\frac{f_{i}\left(x^{*}\right)}{g_{i}\left(x^{*}\right)}\right) \nabla^{2} g_{i}\left(x^{*}\right) z\right], \zeta\left(x, x^{*}\right)\right\rangle \\
& -\frac{1}{2}\left\langle\sum_{i=1}^{p} u_{i}^{*}\left[\nabla^{2} f_{i}\left(x^{*}\right) z-\left(\frac{f_{i}\left(x^{*}\right)}{g_{i}\left(x^{*}\right)}\right) \nabla^{2} g_{i}\left(x^{*}\right) z\right], z\right\rangle \\
\geq & \bar{\rho}\left(x, x^{*}\right)\left\|\theta\left(x, x^{*}\right)\right\|^{2} \geq-\rho\left(x, x^{*}\right)\left\|\theta\left(x, x^{*}\right)\right\|^{2} .
\end{aligned}
$$

Since $\rho\left(x, x^{*}\right) \geq 0$, applying the hybrid $(\Phi, \rho, \eta, \zeta, \theta)$-pseudo-invexity at $x^{*}$ to (3.6) and assumptions on $\Phi$, we have

$$
\Phi\left(\sum_{i=1}^{p} u_{i}^{*}\left[f_{i}(x)-\left(\frac{f_{i}\left(x^{*}\right)}{g_{i}\left(g^{*}\right)}\right) g_{i}(x)\right]-\Sigma_{i=1}^{p} u_{i}^{*}\left[f_{i}\left(x^{*}\right)-\left(\frac{f_{i}\left(x^{*}\right)}{g_{i}\left(x^{*}\right)}\right) g_{i}\left(x^{*}\right)\right]\right) \geq 0,
$$

Stat., Optim. Inf. Comput. Vol. 2, December 2014. 
which implies

$$
\begin{gathered}
\Sigma_{i=1}^{p} u_{i}^{*}\left[f_{i}(x)-\left(\frac{f_{i}\left(x^{*}\right)}{g_{i}\left(x^{*}\right)}\right) g_{i}(x)\right] \\
\left.\geq \quad \sum_{i=1}^{p} u_{i}^{*}\left[f_{i}\left(x^{*}\right)-\left(\frac{f_{i}\left(x^{*}\right)}{g_{i}\left(x^{*}\right)}\right) g_{i}\left(x^{*}\right)\right]\right)=0 .
\end{gathered}
$$

Thus, we have

$$
\Sigma_{i=1}^{p} u_{i}^{*}\left[f_{i}(x)-\left(\frac{f_{i}\left(x^{*}\right)}{g_{i}\left(x^{*}\right)}\right) g_{i}(x)\right] \geq 0 .
$$

Since $u_{i}^{*}>0$ for each $i \in\{1, \cdots, p\}$, we conclude that there does not exist an $x \in Q$ such that

$$
\begin{gathered}
\frac{f_{i}(x)}{g_{i}(x)}-\left(\frac{f_{i}\left(x^{*}\right)}{g_{i}\left(x^{*}\right)}\right) \leq 0 \forall i=1, \cdots, p, \\
\frac{f_{j}(x)}{g_{j}(x)}-\left(\frac{f_{j}\left(x^{*}\right)}{g_{j}\left(x^{*}\right)}\right)<0 \text { for some } j \in\{1, \cdots, p\} .
\end{gathered}
$$

Hence, $x^{*}$ is an efficient solution to $(\mathrm{P})$.

Next, if (ii) holds, and if $x \in Q$, then it follows from (3.1) and (3.2) that

$$
\begin{aligned}
& \left\langle\sum_{i=1}^{p} u_{i}^{*}\left[\nabla f_{i}\left(x^{*}\right)-\left(\frac{f_{i}\left(x^{*}\right)}{g_{i}\left(x^{*}\right)}\right) \nabla g_{i}\left(x^{*}\right)\right], \eta\left(x, x^{*}\right)\right\rangle \\
& +\left\langle\sum_{i=1}^{p} u_{i}^{*}\left[\nabla^{2} f_{i}\left(x^{*}\right) z-\left(\frac{f_{i}\left(x^{*}\right)}{g_{i}\left(x^{*}\right)}\right) \nabla^{2} g_{i}\left(x^{*}\right) z\right], \zeta\left(x, x^{*}\right)\right\rangle \\
& -\frac{1}{2}\left\langle\sum_{i=1}^{p} u_{i}^{*}\left[\nabla^{2} f_{i}\left(x^{*}\right) z-\left(\frac{f_{i}\left(x^{*}\right)}{g_{i}\left(x^{*}\right)}\right) \nabla^{2} g_{i}\left(x^{*}\right) z\right], z\right\rangle \\
& +\left\langle\sum_{j=1}^{m} v_{j}^{*} \nabla H_{j}\left(x^{*}\right), \eta\left(x, x^{*}\right)\right\rangle+\left\langle\Sigma_{j=1}^{m} v_{j}^{*} \nabla^{2} H_{j}\left(x^{*}\right) z, \zeta\left(x, x^{*}\right)\right\rangle \\
& -\frac{1}{2}\left\langle\sum_{j=1}^{m} v_{j}^{*} \nabla^{2} H_{j}\left(x^{*}\right) z, z\right\rangle \geq 0 .
\end{aligned}
$$

Since $v^{*} \geq 0, x \in Q$ and (3.3) holds, we have

$$
\Sigma_{j=1}^{m} v_{j}^{*} H_{j}(x) \leq 0=\Sigma_{j=1}^{m} v_{j}^{*} H_{j}\left(x^{*}\right),
$$

which results (using assumptions on $\tilde{\Phi}$ ) in

$$
\tilde{\Phi}\left(\sum_{j=1}^{m} v_{j}^{*} H_{j}(x)-\Sigma_{j=1}^{m} v_{j}^{*} H_{j}\left(x^{*}\right)\right) \leq 0 .
$$

Stat., Optim. Inf. Comput. Vol. 2, December 2014. 
Now, in light of the strictly hybrid $(\tilde{\Phi}, \bar{\rho}, \eta, \zeta, \theta)$ - quasi-invexity of $B_{j}\left(., v^{*}\right)$ at $x^{*}$, we find

$$
\begin{aligned}
& \left\langle\sum_{j=1}^{m} v_{j}^{*} \nabla H_{j}\left(x^{*}\right), \eta\left(x, x^{*}\right)\right\rangle+\left\langle\sum_{j=1}^{m} v_{j}^{*} \nabla^{2} H_{j}\left(x^{*}\right) z, \zeta\left(x, x^{*}\right)\right\rangle \\
- & \frac{1}{2}\left\langle\Sigma_{j=1}^{m} v_{j}^{*} \nabla^{2} H_{j}\left(x^{*}\right) z, z\right\rangle+\bar{\rho}\left(x, x^{*}\right)\left\|\theta\left(x, x^{*}\right)\right\|^{2}<0 .
\end{aligned}
$$

It follows from (3.8) and (3.9) that

$$
\begin{aligned}
& \left\langle\sum_{i=1}^{p} u_{i}^{*}\left[\nabla f_{i}\left(x^{*}\right)-\left(\frac{f_{i}\left(x^{*}\right)}{g_{i}\left(x^{*}\right)}\right) \nabla g_{i}\left(x^{*}\right)\right], \eta\left(x, x^{*}\right)\right\rangle \\
+ & \left\langle\sum_{i=1}^{p} u_{i}^{*}\left[\nabla^{2} f_{i}\left(x^{*}\right) z-\left(\frac{f_{i}\left(x^{*}\right)}{g_{i}\left(x^{*}\right)}\right) \nabla^{2} g_{i}\left(x^{*}\right) z\right], \zeta\left(x, x^{*}\right)\right\rangle \\
& -\frac{1}{2}\left\langle\sum_{i=1}^{p} u_{i}^{*}\left[\nabla^{2} f_{i}\left(x^{*}\right) z-\left(\frac{f_{i}\left(x^{*}\right)}{g_{i}\left(x^{*}\right)}\right) \nabla^{2} g_{i}\left(x^{*}\right) z\right], z\right\rangle \\
>\quad & \bar{\rho}\left(x, x^{*}\right)\left\|\theta\left(x, x^{*}\right)\right\|^{2}>-\rho\left(x, x^{*}\right)\left\|\theta\left(x, x^{*}\right)\right\|^{2} .
\end{aligned}
$$

As a result, since $\rho\left(x, x^{*}\right) \geq 0$, applying the prestrictly hybrid $(\Phi, \rho, \eta, \zeta, \theta)$-pseudo-invexity at $x^{*}$ to (3.10) and assumptions on $\Phi$, we have

$$
\Phi\left(\Sigma_{i=1}^{p} u_{i}^{*}\left[f_{i}(x)-\left(\frac{f_{i}\left(x^{*}\right)}{g_{i}\left(g^{*}\right)}\right) g_{i}(x)\right]-\Sigma_{i=1}^{p} u_{i}^{*}\left[f_{i}\left(x^{*}\right)-\left(\frac{f_{i}\left(x^{*}\right)}{g_{i}\left(x^{*}\right)}\right) g_{i}\left(x^{*}\right)\right]\right) \geq 0,
$$

which implies

$$
\begin{gathered}
\sum_{i=1}^{p} u_{i}^{*}\left[f_{i}(x)-\left(\frac{f_{i}\left(x^{*}\right)}{g_{i}\left(x^{*}\right)}\right) g_{i}(x)\right] \\
\left.\geq \quad \Sigma_{i=1}^{p} u_{i}^{*}\left[f_{i}\left(x^{*}\right)-\left(\frac{f_{i}\left(x^{*}\right)}{g_{i}\left(x^{*}\right)}\right) g_{i}\left(x^{*}\right)\right]\right)=0 .
\end{gathered}
$$

Thus, we have

$$
\Sigma_{i=1}^{p} u_{i}^{*}\left[f_{i}(x)-\left(\frac{f_{i}\left(x^{*}\right)}{g_{i}\left(x^{*}\right)}\right) g_{i}(x)\right] \geq 0 .
$$

Since $u_{i}^{*}>0$ for each $i \in\{1, \cdots, p\}$, we conclude that there does not exist an $x \in Q$ such that

$$
\begin{gathered}
\frac{f_{i}(x)}{g_{i}(x)}-\left(\frac{f_{i}\left(x^{*}\right)}{g_{i}\left(x^{*}\right)}\right) \leq 0 \forall i=1, \cdots, p, \\
\frac{f_{j}(x)}{g_{j}(x)}-\left(\frac{f_{j}\left(x^{*}\right)}{g_{j}\left(x^{*}\right)}\right)<0 \text { for some } j \in\{1, \cdots, p\} .
\end{gathered}
$$

Stat., Optim. Inf. Comput. Vol. 2, December 2014. 
Hence, $x^{*}$ is an efficient solution to (P). The proof applying (iii) is similar to that of (ii), and we just need to include the proof using (iv) as follows: since $x \in Q$, it follows that $H_{j}(x) \leq H_{j}\left(x^{*}\right)$, which implies $\bar{\Phi}\left(H_{j}(x)-H_{j}\left(x^{*}\right)\right) \leq 0$. Then applying the hybrid $\left(\bar{\Phi}, \rho_{3}, \eta, \zeta, \theta\right)$-quasi-invexity of $H_{j}$ at $x^{*}$ and $v^{*} \in R_{+}^{m}$, we have

$$
\begin{aligned}
& \left\langle\sum_{j=1}^{m} v_{j}^{*} \nabla H_{j}\left(x^{*}\right), \eta\left(x, x^{*}\right)\right\rangle+\left\langle\sum_{j=1}^{m} v_{j}^{*} \nabla^{2} H_{j}\left(x^{*}\right) z, \zeta\left(x, x^{*}\right)\right\rangle \\
& -\frac{1}{2}\left\langle\sum_{j=1}^{m} v_{j}^{*} \nabla^{2} H_{j}\left(x^{*}\right) z, z\right\rangle+\sum_{j=1}^{m} v_{j}^{*} \rho_{3}\left(x, x^{*}\right)\left\|\theta\left(x, x^{*}\right)\right\|^{2} \leq 0 .(3.1
\end{aligned}
$$

Since $u^{*} \geq 0$ and $\frac{f_{i}\left(x^{*}\right)}{g_{i}\left(x^{*}\right)} \geq 0$, it follows from the hybrid $\left(\Phi, \rho_{1}, \eta, \zeta, \theta\right)$-invexity and $\left(\Phi, \rho_{2}, \eta, \zeta, \theta\right)$ - invexity assumptions on $f_{i}$ and $g_{i}$, respectively, that

$$
\begin{aligned}
& \Phi\left(\sum_{i=1}^{p} u_{i}^{*}\left[f_{i}(x)-\left(\frac{f_{i}\left(x^{*}\right)}{g_{i}\left(x^{*}\right)}\right) g_{i}(x)\right]\right) \\
= & \Phi\left(\sum_{i=1}^{p} u_{i}^{*}\left\{\left[f_{i}(x)-f_{i}\left(x^{*}\right)\right]-\left(\frac{f_{i}\left(x^{*}\right)}{g_{i}\left(x^{*}\right)}\right)\left[g_{i}(x)-g_{i}\left(x^{*}\right)\right]\right\}\right) \\
\geq \quad & \sum_{i=1}^{p} u_{i}^{*}\left\{\left\langle\nabla f_{i}\left(x^{*}\right)-\left(\frac{f_{i}\left(x^{*}\right)}{g_{i}\left(x^{*}\right)}\right) \nabla g_{i}\left(x^{*}\right), \eta\left(x, x^{*}\right)\right\rangle\right\} \\
& +\left\langle\sum_{i=1}^{p} u_{i}^{*}\left[\nabla^{2} f_{i}\left(x^{*}\right) z-\left(\frac{f_{i}\left(x^{*}\right)}{g_{i}\left(x^{*}\right)}\right) \nabla^{2} g_{i}\left(x^{*}\right) z, \zeta\left(x, x^{*}\right)\right\rangle\right] \\
& -\frac{1}{2}\left\langle\Sigma_{i=1}^{p} u_{i}^{*}\left[\nabla^{2} f_{i}\left(x^{*}\right) z-\left(\frac{f_{i}\left(x^{*}\right)}{g_{i}\left(x^{*}\right)}\right) \nabla^{2} g_{i}\left(x^{*}\right) z, z\right\rangle\right] \\
& +\sum_{i=1}^{p} u_{i}^{*}\left[\rho_{1}\left(x, x^{*}\right)+\phi\left(x^{*}\right) \rho_{2}\left(x, x^{*}\right)\right]\left\|\theta\left(x, x^{*}\right)\right\|^{2} \\
\geq \quad & -\left[\left\langle\sum_{j=1}^{m} v_{j}^{*} \nabla H_{j}\left(x^{*}\right), \eta\left(x, x^{*}\right)\right\rangle+\left\langle\Sigma_{j=1}^{m} v_{j}^{*} \nabla^{2} H_{j}\left(x^{*}\right) z, \zeta\left(x, x^{*}\right)\right\rangle\right. \\
& \left.-\frac{1}{2}\left\langle\sum_{j=1}^{m} v_{j}^{*} \nabla^{2} H_{j}\left(x^{*}\right) z, z\right\rangle\right]+\Sigma_{i=1}^{p} u_{i}^{*}\left[\rho_{1}\left(x, x^{*}\right)+\phi\left(x^{*}\right) \rho_{2}\left(x, x^{*}\right)\right]\left\|\theta\left(x, x^{*}\right)\right\|^{2} \\
\geq & \left(\sum_{j=1}^{m} v_{j}^{*} \rho_{3}\left(x, x^{*}\right)+\Sigma_{i=1}^{p} u_{i}^{*}\left[\rho_{1}\left(x, x^{*}\right)+\phi\left(x^{*}\right) \rho_{2}\left(x, x^{*}\right)\right]\right)\left\|\theta\left(x, x^{*}\right)\right\|^{2} \\
= & \left(\sum_{j=1}^{m} v_{j}^{*} \rho_{3}\left(x, x^{*}\right)+\rho^{*}\right)\left\|\theta\left(x, x^{*}\right)\right\|^{2} \\
\geq & 0,
\end{aligned}
$$

where $\phi\left(x^{*}\right)=\frac{f_{i}\left(x^{*}\right)}{g_{i}\left(x^{*}\right)} \quad$ and $\quad \rho^{*}=\Sigma_{i=1}^{p} u_{i}^{*}\left(\rho_{1}\left(x, x^{*}\right)+\phi\left(x^{*}\right) \rho_{2}\left(x, x^{*}\right)\right)$. This implies that

$$
\Phi\left(\sum_{i=1}^{p} u_{i}^{*}\left[f_{i}(x)-\left(\frac{f_{i}\left(x^{*}\right)}{g_{i}\left(x^{*}\right)}\right) g_{i}(x)\right]\right) \geq 0 .
$$

\section{Some Specializations}

First, if we consider when $\zeta\left(x, x^{*}\right)=\eta\left(x, x^{*}\right)$ in Theorem 3.1, which means, 
hybrid $(\Phi, \rho, \eta, \theta)$ - invexities, we have the following result which generalizes (Zalmai [35], Theorem 3.2).

Theorem 3.2. Let $x^{*} \in Q$, let $f_{i}, g_{i}$ for $i \in\{1, \cdots, p\}$ with $\frac{f_{i}\left(x^{*}\right)}{g_{i}\left(x^{*}\right)} \geq 0, g_{i}\left(x^{*}\right)>0$ and $H_{j}$ for $j \in\{1, \cdots, m\}$ be twice continuously differentiable at $x^{*} \in Q$, and let there exist $u^{*} \in U=\left\{u \in \Re^{p}: u>0, \Sigma_{i=1}^{p} u_{i}=1\right\}$ and $v^{*} \in \Re_{+}^{m}$ such that

$$
\begin{aligned}
& \sum_{i=1}^{p} u_{i}^{*}\left[\nabla f_{i}\left(x^{*}\right)-\left(\frac{f_{i}\left(x^{*}\right)}{g_{i}\left(x^{*}\right)}\right) \nabla g_{i}\left(x^{*}\right)\right]+\sum_{j=1}^{m} v_{j}^{*} \nabla H_{j}\left(x^{*}\right)=0 \\
& \left\langle\left[\sum_{i=1}^{p} u_{i}^{*}\left[\nabla^{2} f_{i}\left(x^{*}\right)-\left(\frac{f_{i}\left(x^{*}\right)}{g_{i}\left(x^{*}\right)}\right) \nabla^{2} g_{i}\left(x^{*}\right)\right]+\sum_{j=1}^{m} v_{j}^{*} \nabla^{2} H_{j}\left(x^{*}\right)\right] z, \eta\left(x, x^{*}\right)\right\rangle \\
& -\frac{1}{2}\left\langle\left[\sum_{i=1}^{p} u_{i}^{*}\left[\nabla^{2} f_{i}\left(x^{*}\right)-\left(\frac{f_{i}\left(x^{*}\right)}{g_{i}\left(x^{*}\right)}\right) \nabla^{2} g_{i}\left(x^{*}\right)\right]\right.\right. \\
& \left.\left.+\sum_{j=1}^{m} v_{j}^{*} \nabla^{2} H_{j}\left(x^{*}\right)\right] z, z\right\rangle \geq 0
\end{aligned}
$$

and

$$
v_{j}^{*} H_{j}\left(x^{*}\right)=0, j \in\{1, \cdots, m\} .
$$

Suppose, in addition, that any one of the following assumptions holds (for $\left.\rho\left(x, x^{*}\right) \geq 0\right)$ :

(i) $E_{i}\left(. ; x^{*}, u^{*}\right) \forall i \in\{1, \cdots, p\}$ are $(\Phi, \rho, \eta, \theta)$-pseudo-invex at $x^{*} . B_{j}\left(., v^{*}\right)$ $\forall j \in\{1, \cdots, m\}$ are $(\Phi, \bar{\rho}, \eta, \theta)$-quasi-invex at $x^{*}$.

(ii) $E_{i}\left(. ; x^{*}, u^{*}\right) \quad \forall i \in\{1, \cdots, p\}$ are prestrictly hybrid $(\Phi, \rho, \eta, \theta)$-pseudoinvex at $x^{*}$ for $\Phi(a) \geq 0 \Rightarrow a \geq 0$, and $B_{j}\left(., v^{*}\right) \forall j \in\{1, \cdots, m\}$ are strictly hybrid $(\tilde{\Phi}, \rho, \eta, \theta)$-quasi-invex at $x^{*}$ for $\tilde{\Phi}$ increasing and $\tilde{\Phi}(0)=0$.

(iii) $E_{i}\left(. ; x^{*}, u^{*}\right) \quad \forall i \in\{1, \cdots, p\}$ are prestrictly hybrid $(\Phi, \rho, \eta, \theta)$-quasiinvex at $x^{*}$ for $\Phi(a) \geq 0 \Rightarrow a \geq 0$, and $B_{j}\left(., v^{*}\right) \forall j \in\{1, \cdots, m\}$ are strictly hybrid $(\tilde{\Phi}, \bar{\rho}, \eta, \theta)$-quasi-invex at $x^{*}$ for $\tilde{\Phi}$ increasing and $\tilde{\Phi}(0)=0$.

(iv) For each $i \in\{1, \cdots, p\}, f_{i}$ is hybrid $\left(\Phi, \rho_{1}, \eta, \theta\right)$-invex and $-g_{i}$ is hybrid $\left(\Phi, \rho_{2}, \eta, \theta\right)$-invex at $x^{*}$ for $\Phi(a) \geq 0 \Rightarrow a \geq 0, H_{j}\left(., v^{*}\right) \forall j \in$ $\{1, \cdots, m\}$ is hybrid $\left(\bar{\Phi}, \rho_{3}, \eta, \theta\right)$-quasi-invex at $x^{*}$ for $\bar{\Phi}$ increasing and $\bar{\Phi}(0)=0$, and $\Sigma_{j=1}^{m} v_{j}^{*} \rho_{3}\left(x, x^{*}\right)+\rho^{*} \geq 0$ for $\rho^{*}=\Sigma_{i=1}^{p} u_{i}^{*}\left(\rho_{1}\left(x, x^{*}\right)+\right.$ $\left.\phi\left(x^{*}\right) \rho_{2}\left(x, x^{*}\right)\right)$ and for $\phi\left(x^{*}\right)=\frac{f_{i}\left(x^{*}\right)}{g_{i}\left(x^{*}\right)}$.

Then $x^{*}$ is an efficient solution to $(\mathrm{P})$. 
Next we consider the case when the functions are first-order differentiable, Theorem 3.1 reduces to the result which is similar to results of Zalmai and Zhang [37].

Theorem 3.3. Let $x^{*} \in Q$, let $f_{i}, g_{i}$ for $i \in\{1, \cdots, p\}$ with $\frac{f_{i}\left(x^{*}\right)}{g_{i}\left(x^{*}\right)} \geq 0, g_{i}\left(x^{*}\right)>0$ and $H_{j}$ for $j \in\{1, \cdots, m\}$ be differentiable at $x^{*} \in Q$, and let there exist $u^{*} \in U=\left\{u \in \Re^{p}: u>0, \Sigma_{i=1}^{p} u_{i}=1\right\}$ and $v^{*} \in \Re_{+}^{m}$ such that

$$
\left\langle\Sigma_{i=1}^{p} u_{i}^{*}\left[\nabla f_{i}\left(x^{*}\right)-\left(\frac{f_{i}\left(x^{*}\right)}{g_{i}\left(x^{*}\right)}\right) \nabla g_{i}\left(x^{*}\right)\right]+\Sigma_{j=1}^{m} v_{j}^{*} \nabla H_{j}\left(x^{*}\right), \eta\left(x, x^{*}\right)\right\rangle \geq 0
$$

and

$$
v_{j}^{*} H_{j}\left(x^{*}\right)=0, j \in\{1, \cdots, m\} .
$$

Suppose, in addition, that any one of the following assumptions holds (for $\left.\rho\left(x, x^{*}\right) \geq 0\right)$ :

(i) $E_{i}\left(. ; x^{*}, u^{*}\right) \forall i \in\{1, \cdots, p\}$ are first-order hybrid $(\Phi, \rho, \eta, \theta)$-pseudoinvex at $x^{*}$ for $\Phi(a) \geq 0 \Rightarrow a \geq 0$, and $B_{j}\left(., v^{*}\right) \forall j \in\{1, \cdots, m\}$ are firstorder hybrid $(\bar{\Phi}, \bar{\rho}, \eta, \theta)$-quasi-invex at $x^{*}$ for $\bar{\Phi}$ increasing and $\bar{\Phi}(0)=0$.

(ii) $E_{i}\left(. ; x^{*}, u^{*}\right) \quad \forall i \in\{1, \cdots, p\} \quad$ are first-order hybrid prestrictly $(\Phi, \rho, \eta, \theta)$-pseudo-invex at $x^{*}$ for $\Phi(a) \geq 0 \Rightarrow a \geq 0$, and $B_{j}\left(., v^{*}\right)$ $\forall j \in\{1, \cdots, m\}$ are first-order strictly hybrid $(\bar{\Phi}, \bar{\rho}, \eta, \theta)$-quasi-invex at $x^{*}$ for $\bar{\Phi}$ increasing and $\bar{\Phi}(0)=0$.

(iii) $E_{i}\left(. ; x^{*}, u^{*}\right) \quad \forall i \in\{1, \cdots, p\} \quad$ are first-order prestrictly hybrid $(\Phi, \rho, \eta, \theta)$-quasi-invex at $x^{*} \quad \Phi(a) \geq 0 \Rightarrow a \geq 0$, and $B_{j}\left(., v^{*}\right)$ $\forall j \in\{1, \cdots, m\}$ are first-order strictly hybrid $(\bar{\Phi}, \bar{\rho}, \eta, \theta)$-quasi-invex at $x^{*}$ for $\bar{\Phi}$ increasing and $\bar{\Phi}(0)=0$.

(iv) For each $i \in\{1, \cdots, p\}, f_{i}$ is first-order hybrid $\left(\Phi, \rho_{1}, \eta, \theta\right)$-invex and $-g_{i}$ is first-order hybrid $\left(\Phi, \rho_{2}, \eta, \theta\right)$-invex at $x^{*}$ for $\Phi(a) \geq 0 \Rightarrow a \geq 0$. $H_{j}\left(., v^{*}\right) \quad \forall j \in\{1, \cdots, m\} \quad$ is hybrid $\left(\bar{\Phi}, \overline{\rho_{3}}, \eta, \theta\right)$-quasi-invex at $x^{*}$, and $\sum_{j=1}^{m} v_{j}^{*} \overline{\rho_{3}}\left(x, x^{*}\right)+\rho^{*} \geq 0$ for $\bar{\Phi}$ increasing and $\bar{\Phi}(0)=0$, $\rho^{*}=\sum_{i=1}^{p} u_{i}^{*}\left(\rho_{1}\left(x, x^{*}\right)+\phi\left(x^{*}\right) \rho_{2}\left(x, x^{*}\right)\right)$ and for $\phi\left(x^{*}\right)=\frac{f_{i}\left(x^{*}\right)}{g_{i}\left(x^{*}\right)}$.

Then $x^{*}$ is an efficient solution to $(\mathrm{P})$.

Proof

Although the proof is similar to that of Theorem 3.1), we include for the sake of the completeness. If we consider (i), then proceeding as in Theorem 3.1 (and using 
the first-order hybrid $(\Phi, \rho, \eta, \theta)$-invexity assumptions instead), we arrive at

$$
\begin{aligned}
& \left\langle\Sigma_{i=1}^{p} u_{i}^{*}\left[\nabla f_{i}\left(x^{*}\right)-\left(\frac{f_{i}\left(x^{*}\right)}{g_{i}\left(x^{*}\right)}\right) \nabla g_{i}\left(x^{*}\right)\right], \eta\left(x, x^{*}\right)\right\rangle \\
\geq & \rho\left(x, x^{*}\right)\left\|\theta\left(x, x^{*}\right)\right\|^{2} .
\end{aligned}
$$

Since $\rho\left(x, x^{*}\right) \geq 0$, applying the hybrid $(\Phi, \rho, \eta, \theta)$-pseudo-invexity at $x^{*}$ to (3.18) and assumptions on $\Phi$, we have

$$
\Phi\left(\Sigma_{i=1}^{p} u_{i}^{*}\left[f_{i}(x)-\left(\frac{f_{i}\left(x^{*}\right)}{g_{i}\left(g^{*}\right)}\right) g_{i}(x)\right]-\Sigma_{i=1}^{p} u_{i}^{*}\left[f_{i}\left(x^{*}\right)-\left(\frac{f_{i}\left(x^{*}\right)}{g_{i}\left(x^{*}\right)}\right) g_{i}\left(x^{*}\right)\right]\right) \geq 0,
$$

which implies

$$
\begin{aligned}
& \sum_{i=1}^{p} u_{i}^{*}\left[f_{i}(x)-\left(\frac{f_{i}\left(x^{*}\right)}{g_{i}\left(x^{*}\right)}\right) g_{i}(x)\right] \\
\geq & \left.\sum_{i=1}^{p} u_{i}^{*}\left[f_{i}\left(x^{*}\right)-\left(\frac{f_{i}\left(x^{*}\right)}{g_{i}\left(x^{*}\right)}\right) g_{i}\left(x^{*}\right)\right]\right) \\
= & 0 .
\end{aligned}
$$

Thus, we have

$$
\Sigma_{i=1}^{p} u_{i}^{*}\left[f_{i}(x)-\left(\frac{f_{i}\left(x^{*}\right)}{g_{i}\left(x^{*}\right)}\right) g_{i}(x)\right] \geq 0 .
$$

Since $u_{i}^{*}>0$ for each $i \in\{1, \cdots, p\}$, we conclude that there does not exist an $x \in Q$ such that

$$
\begin{gathered}
\frac{f_{i}(x)}{g_{i}(x)}-\left(\frac{f_{i}\left(x^{*}\right)}{g_{i}\left(x^{*}\right)}\right) \leq 0 \forall i=1, \cdots, p, \\
\frac{f_{j}(x)}{g_{j}(x)}-\left(\frac{f_{j}\left(x^{*}\right)}{g_{j}\left(x^{*}\right)}\right)<0 \text { for some } j \in\{1, \cdots, p\} .
\end{gathered}
$$

Hence, $x^{*}$ is an efficient solution to $(\mathrm{P})$.

The proofs for (ii)-(iv) are similar to that of Theorem 3.1.

Next, we note that Theorem 3.1 can be specialized to the context of second order $(\rho, \eta, \theta)$ - invexities as follows:

Theorem 3.4. Let $x^{*} \in Q$. Let $f_{i}, g_{i}$ for $i \in\{1, \cdots, p\}$ with $\frac{f_{i}\left(x^{*}\right)}{g_{i}\left(x^{*}\right)} \geq 0, g_{i}\left(x^{*}\right)>0$ and $H_{j}$ for $j \in\{1, \cdots, m\}$ be twice continuously differentiable at $x^{*} \in Q$, and let there exist $u^{*} \in U=\left\{u \in \Re^{p}: u>0, \Sigma_{i=1}^{p} u_{i}=1\right\}$ and $v^{*} \in \Re_{+}^{m}$ such that

$$
\sum_{i=1}^{p} u_{i}^{*}\left[\nabla f_{i}\left(x^{*}\right)-\left(\frac{f_{i}\left(x^{*}\right)}{g_{i}\left(x^{*}\right)}\right) \nabla g_{i}\left(x^{*}\right)\right]+\sum_{j=1}^{m} v_{j}^{*} \nabla H_{j}\left(x^{*}\right)=0
$$




$$
\left\langle\eta\left(x, x^{*}\right),\left[\sum_{i=1}^{p} u_{i}^{*}\left[\nabla^{2} f_{i}\left(x^{*}\right)-\left(\frac{f_{i}\left(x^{*}\right)}{g_{i}\left(x^{*}\right)}\right) \nabla^{2} g_{i}\left(x^{*}\right)\right]+\sum_{j=1}^{m} v_{j}^{*} \nabla^{2} H_{j}\left(x^{*}\right)\right] z\right\rangle \geq 0,
$$

and

$$
v_{j}^{*} H_{j}\left(x^{*}\right)=0, j \in\{1, \cdots, m\} .
$$

Suppose, in addition, that any one of the following assumptions holds (for $\left.\rho\left(x, x^{*}\right) \geq 0\right)$ :

(i) $E_{i}\left(. ; x^{*}, u^{*}\right) \forall i \in\{1, \cdots, p\}$ are hybrid $(\rho, \eta, \theta)$-pseudo-invex at $x^{*}$, and $B_{j}\left(., v^{*}\right) \forall j \in\{1, \cdots, m\}$ are hybrid $(\rho, \eta, \theta)$-quasi-invex at $x^{*}$.

(ii) $E_{i}\left(. ; x^{*}, u^{*}\right) \forall i \in\{1, \cdots, p\}$ are prestrictly hybrid $(\rho, \eta, \theta)$-pseudo-invex at $x^{*}$, and $B_{j}\left(., v^{*}\right) \forall j \in\{1, \cdots, m\}$ are hybrid $(\rho, \eta, \theta)$-strictly-quasiinvex at $x^{*}$.

(iii) $E_{i}\left(. ; x^{*}, u^{*}\right) \forall i \in\{1, \cdots, p\}$ are strictly hybrid $(\rho, \eta, \theta)$-pseudo-invex at $x^{*}$, and $B_{j}\left(., v^{*}\right) \forall j \in\{1, \cdots, m\}$ are strictly hybrid $(\rho, \eta, \theta)$-quasi-invex at $x^{*}$.

(iv) For each $i \in\{1, \cdots, p\}, \quad f_{i}$ is hybrid $\left(\rho_{1}, \eta, \theta\right)$-invex and $-g_{i}$ is $\left(\rho_{2}, \eta, \theta\right)$-invex at $x^{*} . H_{j}\left(., v^{*}\right) \quad \forall j \in\{1, \cdots, m\}$ is hybrid $\left(\rho_{3}, \eta, \theta\right)$-quasi-invex at $x^{*}, \quad$ and $\sum_{j=1}^{m} v_{j}^{*} \rho_{3}\left(x, x^{*}\right)+\rho^{*} \geq 0$ for $\rho^{*}=\sum_{i=1}^{p} u_{i}^{*}\left(\rho_{1}\left(x, x^{*}\right)+\phi\left(x^{*}\right) \rho_{2}\left(x, x^{*}\right)\right)$ and for $\phi\left(x^{*}\right)=\frac{f_{i}\left(x^{*}\right)}{g_{i}\left(x^{*}\right)}$.

Then $x^{*}$ is an efficient solution to $(\mathrm{P})$.

\section{Proof}

The proof is similar to that of Theorem 3.1 based on the hybrid $(\rho, \eta, \theta)$ - invexity assumptions.

We observe that Theorem 3.1 can be further generalized to the case of the $\epsilon$-efficient conditions based on the hybrid $(\Phi, \rho, \eta, \zeta, \theta)$-invexity frameworks. As a matter of fact, we generalize the $\epsilon$-efficient solvability conditions for problem $(P)$ based on the work of Verma [22], and Kim, Kim and Lee [6], where they have investigated the $\epsilon$-efficiency as well as the weak $\epsilon$-efficiency conditions for multiobjective fractional programming problems under constraint qualifications. To the best of our knowledge, the results established in this communication (Theorem 3.1 and Theorem 3.5) generalize and unify most of the results on the multiobjective fractional programming to the context of the generalized invexities in the literature. We recall some auxiliary concepts (for the hybrid $(\Phi, \rho, \eta, \zeta, \theta)$-invexity) crucial to the problem on hand. 
Definition 3.1. A point $x^{*} \in Q$ is an $\epsilon$-efficient solution to (P) if there does not exist an $x \in Q$ such that

$$
\begin{gathered}
\frac{f_{i}(x)}{g_{i}(x)} \leq \frac{f_{i}\left(x^{*}\right)}{g_{i}\left(x^{*}\right)}-\epsilon_{i} \forall i=1, \cdots, p, \\
\frac{f_{j}(x)}{g_{j}(x)}<\frac{f\left({ }_{j} x^{*}\right)}{g_{j}\left(x^{*}\right)}-\epsilon_{j} \text { for some } j \in\{1, \cdots, p\},
\end{gathered}
$$

where $\epsilon_{i}=\left(\epsilon_{1}, \cdots, \epsilon_{p}\right)$ is with $\epsilon_{i} \geq 0$ for $i=1, \cdots, p$.

For $\epsilon=0$, Definition 3.1 reduces to the case that $x^{*} \in Q$ is an efficient solution to (P).

Next, we start with real-valued functions $E_{i}\left(., x^{*}, u^{*}\right)$ and $B_{j}(., v)$ defined by

$$
E_{i}\left(x, x^{*}, u^{*}\right)=u_{i}\left[f_{i}(x)-\left(\frac{f_{i}\left(x^{*}\right)}{g_{i}\left(x^{*}\right)}-\epsilon_{i}\right) g_{i}(x)\right], i \in\{1, \cdots, p\}
$$

and

$$
B_{j}(., v)=v_{j} H_{j}(x), j=1, \cdots, m
$$

Theorem 3.5. Let $x^{*} \in Q$, let $f_{i}, g_{i}$ for $i \in\{1, \cdots, p\}$ with $f_{i}\left(x^{*}\right) \geq \epsilon_{i} g_{i}\left(x^{*}\right)$, $g_{i}\left(x^{*}\right)>0$ and $H_{j}$ for $j \in\{1, \cdots, m\}$ be twice continuously differentiable at $x^{*} \in Q$, and let there exist $u^{*} \in U=\left\{u \in \Re^{p}: u>0, \Sigma_{i=1}^{p} u_{i}=1\right\}, v^{*} \in \Re_{+}^{m}$ and $z \in \Re^{n}$ such that

$$
\begin{aligned}
& \Sigma_{i=1}^{p} u_{i}^{*}\left[\nabla f_{i}\left(x^{*}\right)-\left(\frac{f_{i}\left(x^{*}\right)}{g_{i}\left(x^{*}\right)}-\epsilon_{i}\right) \nabla g_{i}\left(x^{*}\right)\right]+\Sigma_{j=1}^{m} v_{j}^{*} \nabla H_{j}\left(x^{*}\right)=0, \\
& \left\langle\left[\sum_{i=1}^{p} u_{i}^{*}\left[\nabla^{2} f_{i}\left(x^{*}\right)-\left(\frac{f_{i}\left(x^{*}\right)}{g_{i}\left(x^{*}\right)}-\epsilon_{i}\right) \nabla^{2} g_{i}\left(x^{*}\right)\right]+\sum_{j=1}^{m} v_{j}^{*} \nabla^{2} H_{j}\left(x^{*}\right)\right] z, \zeta\left(x, x^{*}\right)\right\rangle \\
& \quad-\frac{1}{2}\left\langle\left[\sum_{i=1}^{p} u_{i}^{*}\left[\nabla^{2} f_{i}\left(x^{*}\right)-\left(\frac{f_{i}\left(x^{*}\right)}{g_{i}\left(x^{*}\right)}-\epsilon_{i}\right) \nabla^{2} g_{i}\left(x^{*}\right)\right]\right.\right. \\
& \left.\left.+\sum_{j=1}^{m} v_{j}^{*} \nabla^{2} H_{j}\left(x^{*}\right)\right] z, z\right\rangle \geq 0,
\end{aligned}
$$

and

$$
v_{j}^{*} H_{j}\left(x^{*}\right)=0, j \in\{1, \cdots, m\} .
$$


Suppose, in addition, that any one of the following assumptions holds (for $\left.\rho\left(x, x^{*}\right) \geq 0\right)$ :

(i) $E_{i}\left(. ; x^{*}, u^{*}\right) \forall i \in\{1, \cdots, p\}$ are hybrid $(\Phi, \rho, \eta, \zeta, \theta)$-pseudo-invex at $x^{*}$ for $\Phi(a) \geq 0 \Rightarrow a \geq 0$, and $B_{j}\left(., v^{*}\right) \quad \forall j \in\{1, \cdots, m\}$ are hybrid $(\tilde{\Phi}, \bar{\rho}, \eta, \zeta, \theta)$-quasi-invex at $x^{*}$ for $\tilde{\Phi}$ increasing and $\tilde{\Phi}(0)=0$.

(ii) $E_{i}\left(. ; x^{*}, u^{*}\right) \forall i \in\{1, \cdots, p\}$ are prestrictly hybrid $(\Phi, \rho, \eta, \zeta, \theta)$-pseudoinvex at $x^{*}$ for $\Phi(a) \geq 0 \Rightarrow a \geq 0$, and $B_{j}\left(., v^{*}\right) \quad \forall j \in\{1, \cdots, m\}$ are strictly hybrid $(\tilde{\Phi}, \bar{\rho}, \eta, \zeta, \theta)$-quasi-invex at $x^{*}$ for $\tilde{\Phi}$ increasing and $\tilde{\Phi}(0)=0$.

(iii) $E_{i}\left(. ; x^{*}, u^{*}\right) \quad \forall i \in\{1, \cdots, p\}$ are strictly hybrid $(\Phi, \rho, \eta, \zeta, \theta)-$ pseudoinvex at $x^{*}$ for $\Phi(a) \geq 0 \Rightarrow a \geq 0$, and $B_{j}\left(., v^{*}\right) \forall j \in\{1, \cdots, m\}$ are strictly hybrid $(\bar{\Phi}, \bar{\rho}, \eta, \zeta, \theta)$-quasi-invex at $x^{*}$ for $\bar{\Phi}$ increasing and $\bar{\Phi}(0)=$ 0 .

(iv) For each $i \in\{1, \cdots, p\}, f_{i}$ is hybrid $\left(\Phi, \rho_{1}, \eta, \zeta, \theta\right)$-invex and $-g_{i}$ is $\left(\Phi, \rho_{2}, \eta, \zeta, \theta\right)$-invex at $x^{*}$ for $\Phi(a) \geq 0 \Rightarrow a \geq 0$, and $H_{j}\left(., v^{*}\right) \forall j \in$ $\{1, \cdots, m\}$ is hybrid $\left(\bar{\Phi}, \rho_{3}, \eta, \zeta, \theta\right)$-quasi-invex at $x^{*}$ for $\bar{\Phi}$ increasing and $\bar{\Phi}(0)=0$ and $\Sigma_{j=1}^{m} v_{j}^{*} \rho_{3}\left(x, x^{*}\right)+\rho^{*} \geq 0$ for $\rho^{*}=\Sigma_{i=1}^{p} u_{i}^{*}\left(\rho_{1}\left(x, x^{*}\right)+\right.$ $\left.\phi\left(x^{*}\right) \rho_{2}\left(x, x^{*}\right)\right)$, where $\phi\left(x^{*}\right)=\frac{f_{i}\left(x^{*}\right)}{g_{i}\left(x^{*}\right)}-\epsilon_{i}$.

Then $x^{*}$ is an $\epsilon$-efficient solution to (P).

\section{Proof}

If (i) holds, and if $x \in Q$, then it follows from (3.23) and 3.24) that

$$
\begin{aligned}
& \left\langle\Sigma_{i=1}^{p} u_{i}^{*}\left[\nabla f_{i}\left(x^{*}\right)-\left(\frac{f_{i}\left(x^{*}\right)}{g_{i}\left(x^{*}\right)}-\epsilon_{i}\right) \nabla g_{i}\left(x^{*}\right)\right], \eta\left(x, x^{*}\right)\right\rangle \\
& +\left\langle\sum_{i=1}^{p} u_{i}^{*}\left[\nabla^{2} f_{i}\left(x^{*}\right) z-\left(\frac{f_{i}\left(x^{*}\right)}{g_{i}\left(x^{*}\right)}-\epsilon_{i}\right) \nabla^{2} g_{i}\left(x^{*}\right) z\right], \zeta\left(x, x^{*}\right)\right\rangle \\
& -\frac{1}{2}\left\langle\sum_{i=1}^{p} u_{i}^{*}\left[\nabla^{2} f_{i}\left(x^{*}\right) z-\left(\frac{f_{i}\left(x^{*}\right)}{g_{i}\left(x^{*}\right)}-\epsilon_{i}\right) \nabla^{2} g_{i}\left(x^{*}\right) z\right], z\right\rangle \\
& +\left\langle\sum_{j=1}^{m} v_{j}^{*} \nabla H_{j}\left(x^{*}\right), \eta\left(x, x^{*}\right)\right\rangle+\left\langle\Sigma_{j=1}^{m} v_{j}^{*} \nabla^{2} H_{j}\left(x^{*}\right) z, \zeta\left(x, x^{*}\right)\right\rangle \\
& -\frac{1}{2}\left\langle\sum_{j=1}^{m} v_{j}^{*} \nabla^{2} H_{j}\left(x^{*}\right) z, z\right\rangle \geq 0 .
\end{aligned}
$$

Since $v^{*} \geq 0, x \in Q$ and (3.25) holds, we have

$$
\Sigma_{j=1}^{m} v_{j}^{*} H_{j}(x) \leq 0=\Sigma_{j=1}^{m} v_{j}^{*} H_{j}\left(x^{*}\right),
$$

which implies

$$
\Sigma_{j=1}^{m} v_{j}^{*} H_{j}(x)-\Sigma_{j=1}^{m} v_{j}^{*} H_{j}\left(x^{*}\right) \leq 0,
$$

Stat., Optim. Inf. Comput. Vol. 2, December 2014. 
so using the assumptions on $\tilde{\Phi}$ it results in

$$
\tilde{\Phi}\left(\Sigma_{j=1}^{m} v_{j}^{*} H_{j}(x)-\Sigma_{j=1}^{m} v_{j}^{*} H_{j}\left(x^{*}\right)\right) \leq 0,
$$

which, in light of the hybrid $(\tilde{\Phi}, \bar{\rho}, \eta, \zeta, \theta)-$ quasi-invexity of $B_{j}\left(., v^{*}\right)$ at $x^{*}$, implies

$$
\begin{gathered}
\left\langle\nabla H_{j}\left(x^{*}\right), \eta\left(x, x^{*}\right)\right\rangle+\left\langle\zeta\left(x, x^{*}\right), \nabla^{2} H_{j}\left(x^{*}\right) z\right\rangle-\frac{1}{2}\left\langle\nabla^{2} H_{j}\left(x^{*}\right) z, z\right\rangle \\
+\bar{\rho}\left(x, x^{*}\right)\left\|\theta\left(x, x^{*}\right)\right\|^{2} \leq 0 .
\end{gathered}
$$

It follows from (3.26) and (3.27) that

$$
\begin{aligned}
& \left\langle\Sigma_{i=1}^{p} u_{i}^{*}\left[\nabla f_{i}\left(x^{*}\right)-\left(\frac{f_{i}\left(x^{*}\right)}{g_{i}\left(x^{*}\right)}-\epsilon_{i}\right) \nabla g_{i}\left(x^{*}\right)\right], \eta\left(x, x^{*}\right)\right\rangle \\
+ & \left\langle\sum_{i=1}^{p} u_{i}^{*}\left[\nabla^{2} f_{i}\left(x^{*}\right) z-\left(\frac{f_{i}\left(x^{*}\right)}{g_{i}\left(x^{*}\right)}-\epsilon_{i}\right) \nabla^{2} g_{i}\left(x^{*}\right) z\right], \zeta\left(x, x^{*}\right)\right\rangle \\
& -\frac{1}{2}\left\langle\sum_{i=1}^{p} u_{i}^{*}\left[\nabla^{2} f_{i}\left(x^{*}\right) z-\left(\frac{f_{i}\left(x^{*}\right)}{g_{i}\left(x^{*}\right)}-\epsilon_{i}\right) \nabla^{2} g_{i}\left(x^{*}\right) z\right], z\right\rangle \\
\geq & \bar{\rho}\left(x, x^{*}\right)\left\|\theta\left(x, x^{*}\right)\right\|^{2} \geq-\rho\left(x, x^{*}\right)\left\|\theta\left(x, x^{*}\right)\right\|^{2} .
\end{aligned}
$$

As a result, since $\rho\left(x, x^{*}\right) \geq 0$, applying the hybrid $(\Phi, \rho, \eta, \zeta, \theta)-$ pseudoinvexity at $x^{*}$ to (3.28) and assumptions on $\Phi$, we have

$$
\begin{aligned}
& \Phi\left(\sum_{i=1}^{p} u_{i}^{*}\left[f_{i}(x)-\left(\frac{f_{i}\left(x^{*}\right)}{g_{i}\left(x^{*}\right)}-\epsilon_{i}\right) g_{i}(x)\right]\right. \\
& \left.-\Sigma_{i=1}^{p} u_{i}^{*}\left[f_{i}\left(x^{*}\right)-\left(\frac{f_{i}\left(x^{*}\right)}{g_{i}\left(x^{*}\right)}-\epsilon_{i}\right) g_{i}\left(x^{*}\right)\right]\right) \geq 0,
\end{aligned}
$$

which implies

$$
\begin{aligned}
& \sum_{i=1}^{p} u_{i}^{*}\left[f_{i}(x)-\left(\frac{f_{i}\left(x^{*}\right)}{g_{i}\left(x^{*}\right)}-\epsilon_{i}\right) g_{i}(x)\right] \\
& \geq \Sigma_{i=1}^{p} u_{i}^{*}\left[f_{i}\left(x^{*}\right)-\left(\frac{f_{i}\left(x^{*}\right)}{g_{i}\left(x^{*}\right)}-\epsilon_{i}\right) g_{i}\left(x^{*}\right)\right] \\
& \geq \sum_{i=1}^{p} u_{i}^{*}\left[f_{i}\left(x^{*}\right)-\left(\frac{f_{i}\left(x^{*}\right)}{g_{i}\left(x^{*}\right)}-\epsilon_{i}\right) g_{i}\left(x^{*}\right)\right] \\
& -\sum_{i=1}^{p} u_{i}^{*} \epsilon_{i} g_{i}\left(x^{*}\right)=0 .
\end{aligned}
$$

Thus, we have

$$
\Sigma_{i=1}^{p} u_{i}^{*}\left[f_{i}(x)-\left(\frac{f_{i}\left(x^{*}\right)}{g_{i}\left(x^{*}\right)}-\epsilon_{i}\right) g_{i}(x)\right] \geq 0 .
$$


Since $u_{i}^{*}>0$ for each $i \in\{1, \cdots, p\}$, we conclude that there does not exist an $x \in Q$ such that

$$
\begin{gathered}
\sum_{i=1}^{p}\left[\frac{f_{i}(x)}{g_{i}(x)}-\left(\frac{f_{i}\left(x^{*}\right)}{g_{i}\left(x^{*}\right)}-\epsilon_{i}\right)\right] \leq 0 \forall i=1, \cdots, p, \\
\sum_{j=1}^{p}\left[\frac{f_{j}(x)}{g_{j}(x)}-\left(\frac{f_{j}\left(x^{*}\right)}{g_{j}\left(x^{*}\right)}-\epsilon_{j}\right)\right]<0 \text { for some } j \in\{1, \cdots, p\} .
\end{gathered}
$$

Hence, $x^{*}$ is an $\epsilon$-efficient solution to (P).

If (ii) holds, and if $x \in Q$, then it follows from (3.23) and (3.24) that

$$
\begin{aligned}
& \left\langle\sum_{i=1}^{p} u_{i}^{*}\left[\nabla f_{i}\left(x^{*}\right)-\left(\frac{f_{i}\left(x^{*}\right)}{g_{i}\left(x^{*}\right)}-\epsilon_{i}\right) \nabla g_{i}\left(x^{*}\right)\right], \eta\left(x, x^{*}\right)\right\rangle \\
& +\left\langle\sum_{i=1}^{p} u_{i}^{*}\left[\nabla^{2} f_{i}\left(x^{*}\right) z-\left(\frac{f_{i}\left(x^{*}\right)}{g_{i}\left(x^{*}\right)}-\epsilon_{i}\right) \nabla^{2} g_{i}\left(x^{*}\right) z\right], \zeta\left(x, x^{*}\right)\right\rangle \\
& -\frac{1}{2}\left\langle\sum_{i=1}^{p} u_{i}^{*}\left[\nabla^{2} f_{i}\left(x^{*}\right) z-\left(\frac{f_{i}\left(x^{*}\right)}{g_{i}\left(x^{*}\right)}-\epsilon_{i}\right) \nabla^{2} g_{i}\left(x^{*}\right) z\right], z\right\rangle \\
& +\left\langle\sum_{j=1}^{m} v_{j}^{*} \nabla H_{j}\left(x^{*}\right), \eta\left(x, x^{*}\right)\right\rangle+\left\langle\Sigma_{j=1}^{m} v_{j}^{*} \nabla^{2} H_{j}\left(x^{*}\right) z, \zeta\left(x, x^{*}\right)\right\rangle \\
& -\frac{1}{2}\left\langle\sum_{j=1}^{m} v_{j}^{*} \nabla^{2} H_{j}\left(x^{*}\right) z, z\right\rangle \geq 0 .
\end{aligned}
$$

Since $v^{*} \geq 0, x \in Q$ and (3.25) holds, we have

$$
\Sigma_{j=1}^{m} v_{j}^{*} H_{j}(x) \leq 0=\Sigma_{j=1}^{m} v_{j}^{*} H_{j}\left(x^{*}\right),
$$

or

$$
\Sigma_{j=1}^{m} v_{j}^{*} H_{j}(x)-\Sigma_{j=1}^{m} v_{j}^{*} H_{j}\left(x^{*}\right) \leq 0,
$$

which implies based on assumptions on $\tilde{\Phi}$ that

$$
\tilde{\Phi}\left(\Sigma_{j=1}^{m} v_{j}^{*} H_{j}(x)-\Sigma_{j=1}^{m} v_{j}^{*} H_{j}\left(x^{*}\right)\right) \leq 0 .
$$

Next, in light of the strict $(\tilde{\Phi}, \bar{\rho}, \eta, \zeta, \theta)$-quasi-invexity of $B_{j}\left(., v^{*}\right)$ at $x^{*}$ with $\tilde{\Phi}$ increasing and $\tilde{\Phi}(0)=0$, we find

$$
\begin{gathered}
\left\langle\nabla H_{j}\left(x^{*}\right), \eta\left(x, x^{*}\right)\right\rangle+\left\langle\zeta\left(x, x^{*}\right), \nabla^{2} H_{j}\left(x^{*}\right) z\right\rangle-\frac{1}{2}\left\langle\nabla^{2} H_{j}\left(x^{*}\right) z, z\right\rangle \\
+\bar{\rho}\left(x, x^{*}\right)\left\|\theta\left(x, x^{*}\right)\right\|^{2}<0 .
\end{gathered}
$$

Stat., Optim. Inf. Comput. Vol. 2, December 2014. 
It follows from (3.30) and (3.31) that

$$
\begin{aligned}
& \left\langle\sum_{i=1}^{p} u_{i}^{*}\left[\nabla f_{i}\left(x^{*}\right)-\left(\frac{f_{i}\left(x^{*}\right)}{g_{i}\left(x^{*}\right)}-\epsilon_{i}\right) \nabla g_{i}\left(x^{*}\right)\right], \eta\left(x, x^{*}\right)\right\rangle \\
+ & \left\langle\sum_{i=1}^{p} u_{i}^{*}\left[\nabla^{2} f_{i}\left(x^{*}\right) z-\left(\frac{f_{i}\left(x^{*}\right)}{g_{i}\left(x^{*}\right)}-\epsilon_{i}\right) \nabla^{2} g_{i}\left(x^{*}\right) z\right], \zeta\left(x, x^{*}\right)\right\rangle \\
& -\frac{1}{2}\left\langle\sum_{i=1}^{p} u_{i}^{*}\left[\nabla^{2} f_{i}\left(x^{*}\right) z-\left(\frac{f_{i}\left(x^{*}\right)}{g_{i}\left(x^{*}\right)}-\epsilon_{i}\right) \nabla^{2} g_{i}\left(x^{*}\right) z\right], z\right\rangle \\
>\quad & \bar{\rho}\left(x, x^{*}\right)\left\|\theta\left(x, x^{*}\right)\right\|^{2}>-\rho\left(x, x^{*}\right)\left\|\theta\left(x, x^{*}\right)\right\|^{2} .
\end{aligned}
$$

As a result, since $\rho\left(x, x^{*}\right) \geq 0$, applying the prestrictly hybrid $(\Phi, \rho, \eta, \zeta, \theta)$-pseudo-invexity at $x^{*}$ to (3.32) and assumptions on $\Phi$, we have

$$
\begin{aligned}
& \Phi\left(\sum_{i=1}^{p} u_{i}^{*}\left[f_{i}(x)-\left(\frac{f_{i}\left(x^{*}\right)}{g_{i}\left(x^{*}\right)}-\epsilon_{i}\right) g_{i}(x)\right]\right. \\
& \left.\left.-\sum_{i=1}^{p} u_{i}^{*}\left[f_{i}\left(x^{*}\right)-\left(\frac{f_{i}\left(x^{*}\right)}{g_{i}\left(x^{*}\right)}\right)-\epsilon_{i}\right) g_{i}\left(x^{*}\right)\right]\right) \geq 0,
\end{aligned}
$$

which implies

$$
\begin{aligned}
& \sum_{i=1}^{p} u_{i}^{*}\left[f_{i}(x)-\left(\frac{f_{i}\left(x^{*}\right)}{g_{i}\left(x^{*}\right)}-\epsilon_{i}\right) g_{i}(x)\right] \\
\geq & \sum_{i=1}^{p} u_{i}^{*}\left[f_{i}\left(x^{*}\right)-\left(\frac{f_{i}\left(x^{*}\right)}{g_{i}\left(x^{*}\right)}-\epsilon_{i}\right) g_{i}\left(x^{*}\right)\right] \\
\geq & \sum_{i=1}^{p} u_{i}^{*}\left[f_{i}\left(x^{*}\right)-\left(\frac{f_{i}\left(x^{*}\right)}{g_{i}\left(x^{*}\right)}-\epsilon_{i}\right) g_{i}\left(x^{*}\right)\right]-\Sigma_{i=1}^{p} u_{i}^{*} \epsilon_{i} g_{i}\left(x^{*}\right) \\
= & 0 .
\end{aligned}
$$

Thus, we have

$$
\sum_{i=1}^{p} u_{i}^{*}\left[f_{i}(x)-\left(\frac{f_{i}\left(x^{*}\right)}{g_{i}\left(x^{*}\right)}-\epsilon_{i}\right) g_{i}(x)\right] \geq 0 .
$$

Since $u_{i}^{*}>0$ for each $i \in\{1, \cdots, p\}$, we conclude that there does not exist an $x \in Q$ such that

$$
\begin{gathered}
\frac{f_{i}(x)}{g_{i}(x)}-\left(\frac{f_{i}\left(x^{*}\right)}{g_{i}\left(x^{*}\right)}-\epsilon_{i}\right) \leq 0 \forall i=1, \cdots, p, \\
\frac{f_{j}(x)}{g_{j}(x)}-\left(\frac{f_{j}\left(x^{*}\right)}{g_{j}\left(x^{*}\right)}-\epsilon_{j}\right)<0 \text { for some } j \in\{1, \cdots, p\} .
\end{gathered}
$$

Hence, $x^{*}$ is an $\epsilon$-efficient solution to $(\mathrm{P})$. 
The proof applying (iii) is similar to that of (ii), and we just prove using (iv) as follows: since $x \in Q$, it follows that $H_{j}(x) \leq H_{j}\left(x^{*}\right)$. Then applying the $\left(\bar{\Phi}, \rho_{3}, \eta, \zeta, \theta\right)$-quasi-invexity of $H_{j}$ at $x^{*}$ and $v^{*} \in R_{+}^{m}$, we have

$$
\begin{aligned}
& \left\langle\Sigma_{j=1}^{m} v_{j}^{*} \nabla H_{j}\left(x^{*}\right), \eta\left(x, x^{*}\right)\right\rangle+\left\langle\zeta\left(x, x^{*}\right), \Sigma_{j=1}^{m} v_{j}^{*} \nabla^{2} H_{j}\left(x^{*}\right) z\right\rangle \\
& -\frac{1}{2}\left\langle\Sigma_{j=1}^{m} v_{j}^{*} \nabla^{2} H_{j}\left(x^{*}\right) z, z\right\rangle \leq-\Sigma_{j=1}^{m} v_{j}^{*} \rho_{3}\left\|\theta\left(x, x^{*}\right)\right\|^{2} .
\end{aligned}
$$

Since $u^{*} \geq 0$ and $f_{i}\left(x^{*}\right) \geq \epsilon_{i} g_{i}\left(x^{*}\right)$, it follows from $\left(\Phi, \rho_{1}, \eta, \zeta, \theta\right)$-invexity and $\left(\Phi, \rho_{2}, \eta, \zeta, \theta\right)$-invexity assumptions that

$$
\begin{aligned}
& \Phi\left(\sum_{i=1}^{p} u_{i}^{*}\left[f_{i}(x)-\left(\frac{f_{i}\left(x^{*}\right)}{g_{i}\left(x^{*}\right)}-\epsilon_{i}\right) g_{i}(x)\right]\right) \\
= & \Phi\left(\sum_{i=1}^{p} u_{i}^{*}\left\{\left[f_{i}(x)-f_{i}\left(x^{*}\right)\right]-\left(\frac{f_{i}\left(x^{*}\right)}{g_{i}\left(x^{*}\right)}-\epsilon_{i}\right)\left[g_{i}(x)-g_{i}\left(x^{*}\right)\right]+\epsilon_{i} g_{i}\left(x^{*}\right)\right\}\right) \\
\geq \quad & \sum_{i=1}^{p} u_{i}^{*}\left\{\left\langle\nabla f_{i}\left(x^{*}\right)-\left(\frac{f_{i}\left(x^{*}\right)}{g_{i}\left(x^{*}\right)}-\epsilon_{i}\right) \nabla g_{i}\left(x^{*}\right), \eta\left(x, x^{*}\right)\right\rangle\right\} \\
& +\left\langle\zeta\left(x, x^{*}\right), \Sigma_{i=1}^{p} u_{i}^{*}\left[\nabla^{2} f_{i}\left(x^{*}\right) z-\left(\frac{f_{i}\left(x^{*}\right)}{g_{i}\left(x^{*}\right)}-\epsilon_{i}\right) \nabla^{2} g_{i}\left(x^{*}\right) z\right\rangle\right] \\
& -\frac{1}{2}\left\langle\Sigma_{i=1}^{p} u_{i}^{*}\left[\nabla^{2} f_{i}\left(x^{*}\right) z-\left(\frac{f_{i}\left(x^{*}\right)}{g_{i}\left(x^{*}\right)}-\epsilon_{i}\right) \nabla^{2} g_{i}\left(x^{*}\right) z, z\right\rangle\right. \\
& +\left[\rho_{1}\left(x, x^{*}\right)+\phi\left(x^{*}\right) \rho_{2}\left(x, x^{*}\right)\right]\left\|\theta\left(x, x^{*}\right)\right\|^{2}+\Sigma_{i=1}^{p} u_{i}^{*} \epsilon_{i} g_{i}\left(x^{*}\right) \\
\geq \quad & -\left[\left\langle\sum_{j=1}^{m} v_{j}^{*} \nabla H_{j}\left(x^{*}\right), \eta\left(x, x^{*}\right)\right\rangle+\left\langle\zeta\left(x, x^{*}\right), \Sigma_{j=1}^{m} v_{j}^{*} \nabla^{2} H_{j}\left(x^{*}\right) z\right\rangle\right. \\
& \left.-\frac{1}{2}\left\langle\sum_{j=1}^{m} v_{j}^{*} \nabla^{2} H_{j}\left(x^{*}\right) z, z\right\rangle\right] \\
& +\Sigma_{i=1}^{p} u_{i}^{*}\left[\rho_{1}\left(x, x^{*}\right)+\phi\left(x^{*}\right) \rho_{2}\left(x, x^{*}\right)\right]\left\|\theta\left(x, x^{*}\right)\right\|^{2}+\Sigma_{i=1}^{p} u_{i}^{*} \epsilon_{i} g_{i}\left(x^{*}\right) \\
\geq \quad & \left(\Sigma_{j=1}^{m} v_{j}^{*} \rho_{3}\left(x, x^{*}\right)+\Sigma_{i=1}^{p} u_{i}^{*}\left[\rho_{1}\left(x, x^{*}\right)+\phi\left(x^{*}\right) \rho_{2}\left(x, x^{*}\right)\right]\right)\left\|\theta\left(x, x^{*}\right)\right\|^{2} \\
& +\sum_{i=1}^{p} u_{i}^{*} \epsilon_{i} g_{i}\left(x^{*}\right) \\
= & \left(\sum_{j=1}^{m} v_{j}^{*} \rho_{3}\left(x, x^{*}\right)+\rho^{*}\right)\left\|\theta\left(x, x^{*}\right)\right\|^{2}+\Sigma_{i=1}^{p} u_{i}^{*} \epsilon_{i} g_{i}\left(x^{*}\right) \\
\geq \quad & \left(\sum_{j=1}^{m} v_{j}^{*} \rho_{3}\left(x, x^{*}\right)+\rho^{*}\right)\left\|\theta\left(x, x^{*}\right)\right\|^{2} \geq 0 .
\end{aligned}
$$

Therefore, we have

$$
\sum_{i=1}^{p} u_{i}^{*}\left[f_{i}(x)-\left(\frac{f_{i}\left(x^{*}\right)}{g_{i}\left(x^{*}\right)}-\epsilon_{i}\right) g_{i}(x)\right] \geq 0 .
$$

Thus, we conclude that there does not exist an $x \in Q$ such that

$$
\sum_{i=1}^{p}\left[\frac{f_{i}(x)}{g_{i}(x)}-\left(\frac{f_{i}\left(x^{*}\right)}{g_{i}\left(x^{*}\right)}-\epsilon_{i}\right)\right] \leq 0 \forall i=1, \cdots, p,
$$

Stat., Optim. Inf. Comput. Vol. 2, December 2014. 


$$
\sum_{j=1}^{p}\left[\frac{f_{j}(x)}{g_{j}(x)}-\left(\frac{f_{j}\left(x^{*}\right)}{g_{j}\left(x^{*}\right)}-\epsilon_{j}\right)\right]<0 \text { for some } j \in\{1, \cdots, p\} .
$$

Hence, $x^{*}$ is an $\epsilon$-efficient solution to $(\mathrm{P})$.

\section{Concluding Remarks}

We established several results on multiobjective fractional programming problems based on the generalized hybrid $(\Phi, \rho, \eta, \zeta, \theta)$-invexities and on efficient solutions to the multiobjective fractional programming problems. We observe that the obtained results in this communication can be generalized to the case of multiobjective fractional programming with generalized hybrid invex functions of higher orders (including the exponential type generalized invexities), for instance, based on the work of Mishra and Rueda [11], Mishra, Laha and Verma [13], and Zalmai and Zhang [38] to the case of the efficiency as well as to the $\epsilon$-efficiency conditions relating to the minimax fractional programming problems involving generalized invex functions. Furthermore, the hybrid $(\Phi, \rho, \eta, \zeta, \theta)$ - invexities can effectively be applied generalizing/unifying the first-order sufficient efficiency condition results [35], first-order parametric duality model results [36] as well as second-order duality model results (Zalmai [37]) on Hanson-Antczak-type generalized V-invex functions in semiinfinite multiobjective fractional programming. Based on new duality models and suitable constraint structures, the weak, strong, and strict converse duality theorems can be established using appropriate hybrid $(\Phi, \rho, \eta, \zeta, \theta)$ - invexities.

\section{Acknowledgment}

The author is greatly indebted to the reviewers for their highly constructive comments and valuable suggestions leading the revised version.

\section{REFERENCES}

1. A. Ben-Israel and B. Mond, What is the invexity? Journal of Australian Mathematical Society Ser. B 28 (1986), 1 - 9.

2. L. Caiping and Y. Xinmin, Generalized $(\rho, \theta, \eta)$-invariant monotonicity and generalized $(\rho, \theta, \eta)$-invexity of non-differentiable functions, Journal of Inequalities and Applications Vol. 2009(2009), Article ID \# 393940, 16 pages.

3. M. A. Hanson, On sufficiency of the Kuhn-Tucker conditions, Journal of Mathematical Analysis and Applications 80 (1981), 545 - 550.

4. V. Jeyakumar, Strong and weak invexity in mathematical programming, Methods Oper. Res. 55 (1985), 109-125. 
5. H. Kawasaki, Second-order necessary conditions of the Kuhn-Tucker type under new constraint qualifications, Journal of Optimization Theory and Applications 57 (2) (1988), $253-264$.

6. M. H. Kim, G. S. Kim and G. M. Lee, On $\epsilon-$ optimality conditions for multiobjective fractional optimization problems, Fixed Point Theory \& Applications 2011:6 doi:10.1186/1687-18122011-6.

7. J. C. Liu, Second order duality for minimax programming, Utilitas Math. 56 (1999), 53 - 63

8. O. L. Mangasarian, Second- and higher-order duality theorems in nonlinear programming, $J$. Math. Anal. Appl. 51 (1975), 607 - 620.

9. S. K. Mishra, Second order generalized invexity and duality in mathematical programming, Optimization 42 (1997), 51 - 69.

10. S. K. Mishra, Second order symmetric duality in mathematical programming with Fconvexity, European J. Oper. Res. 127 (2000), 507 - 518.

11. S. K. Mishra and N. G. Rueda, Higher-order generalized invexity and duality in mathematical programming, J. Math. Anal. Appl. 247 (2000), 173 - 182.

12. S. K. Mishra and N. G. Rueda, Second-order duality for nondifferentiable minimax programming involving generalized type I functions, J. Optim. Theory Appl. 130 (2006), 477 -486 .

13. S. K. Mishra, V. Laha and R. U. Verma, Generalized vector variational-like inequalities and nonsmooth vector optimization of radially continuous functions, Advances in Nonlinear Variational Inequalities 14 (2)(2011), 1 - 18.

14. B. Mond and T. Weir, Generalized convexity and higher-order duality, J. Math. Sci. 16-18 (1981-1983), 74 - 94.

15. B. Mond and J. Zhang, Duality for multiobjective programming involving second-order Vinvex functions, in Proceedings of the Optimization Miniconference II (B. M. Glover and V. Jeyakumar, eds.), University of New South Wales, Sydney, Australia, 1995, pp. 89 - 100.

16. B. Mond and J. Zhang, Higher order invexity and duality in mathemaical programming, in Generalized Convexity, Generalized Monotonicity : Recent Results (J. P. Crouzeix, et al., eds.), Kluwer Academic Publishers, printed in the Netherlands, 1998, pp. 357 - 372.

17. R. B. Patel, Second order duality in multiobjective fractional programming, Indian J. Math. 38 (1997), 39 - 46.

18. M. K. Srivastava and M. Bhatia, Symmetric duality for multiobjective programming using second order $(F, \rho)$-convexity, Opsearch 43 (2006), 274 - 295.

19. K. K. Srivastava and M. G. Govil, Second order duality for multiobjective programming involving $(F, \rho, \sigma)$-type I functions, Opsearch 37 (2000), 316 - 326.

20. S. K. Suneja, C. S. Lalitha, and S. Khurana, Second order symmetric duality in multiobjective programming, European J. Oper. Res. 144 (2003), 492 - 500.

21. M. N. Vartak and I. Gupta, Duality theory for fractional programming problems under $\eta$ convexity, Opsearch 24 (1987), 163 - 174.

22. R. U. Verma, Weak $\epsilon-$ efficiency conditions for multiobjective fractional programming, Applied Mathematics and Computation 219 (2013), 6819 - 6827.

23. R. U. Verma, New $\epsilon$-optimality conditions for multiobjective fractional subset programming problems, Transactions on Mathematical Programming and Applications 1 (1)(2013), 69 - 89.

24. R. U. Verma, Second-order $(\Phi, \eta, \rho, \theta)$-invexities and parameter-free $\epsilon$-efficiency conditions for multiobjective discrete minmax fractional programming problems, Advances in Nonlinear Variational Inequalities 17 (1)(2014), 27 - 46.

25. X. M. Yang, Second order symmetric duality for nonlinear programs, Opsearch 32 (1995), $205-209$.

26. X. M. Yang, On second order symmetric duality in nondifferentiable multiobjective programming, J. Ind. Manag. Optim. 5 (2009), 697 - 703.

27. X. M. Yang and S. H. Hou, Second-order symmetric duality in multiobjective programming, Appl. Math. Lett. 14 (2001), 587 - 592.

28. X. M. Yang, K. L. Teo and X. Q. Yang, Higher-order generalized convexity and duality in nondifferentiable multiobjective mathematical programming, J. Math. Anal. Appl. 29 (2004), $48-55$.

Stat., Optim. Inf. Comput. Vol. 2, December 2014. 
29. X. M. Yang, X. Q. Yang and K. L. Teo, Nondifferentiable second order symmetric duality in mathematical programming with F-convexity, European J. Oper. Res. 144 (2003), 554 - 559.

30. X. M. Yang, X. Q. Yang and K. L. Teo, Huard type second-order converse duality for nonlinear programming, Appl. Math. Lett. 18 (2005), 205 - 208.

31. X. M. Yang, X. Q. Yang and K. L. Teo, Higher-order symmetric duality in multiobjective programming with invexity, J. Ind. Manag. Optim. 4 (2008), 385 - 391.

32. X. M. Yang, X. Q. Yang, K. L. Teo and S. H. Hou, Second order duality for nonlinear programming, Indian J. Pure Appl. Math. 35 (2004), 699 - 708.

33. K. Yokoyama, Epsilon approximate solutions for multiobjective programming problems, Journal of Mathematical Analysis and Applications 203 (1) (1996), 142 - 149.

34. G. J. Zalmai, Global parametric sufficient optimality conditions for discrete minmax fractional programming problems containing generalized $(\theta, \eta, \rho)$-V-invex functions and arbitrary norms Journal of Applied Mathematics \& Computing 23 (1-2) (2007), 1 - 23.

35. G. J. Zalmai, Hanson-Antczak-type generalized $(\alpha, \beta, \gamma, \xi, \eta, \rho, \theta)$-V-invex functions in semiinfinite multiobjective fractional programming, I : Sufficient efficiency conditions, Advances in Nonlinear Variational Inequalities 16 (1) (2013), 91 - 114.

36. G. J. Zalmai, Hanson-Antczak-type generalized $(\alpha, \beta, \gamma, \xi, \eta, \rho, \theta)$-V-invex functions in semiinfinite multiobjective fractional programming, II : First-order parametric duality models, Advances in Nonlinear Variational Inequalities 16 (2) (2013), 61 - 90.

37. G. J. Zalmai, Hanson-Antczak-type generalized $(\alpha, \beta, \gamma, \xi, \eta, \rho, \theta)$-V-invex functions in semiinfinite multiobjective fractional programming, III: Second-order parametric duality models, Advances in Nonlinear Variational Inequalities 16 (2) (2013), 91 - 126.

38. G. J. Zalmai and Q. Zhang, Global nonparametric sufficient optimality conditions for semiinfinite discrete minmax fractional programming problems involving generalized $(\rho, \theta)$-invex functions, Numerical Functional Analysis and Optimization 28(1-2) (2007), 173 - 209.

39. J. Zhang and B. Mond, Second order b-invexity and duality in mathematical programming, Utilitas Math. 50 (1996), 19 - 31.

40. J. Zhang and B. Mond, Second order duality for multiobjective nonlinear programming involving generalized convexity, in Proceedings of the Optimization Miniconference III (B. M. Glover, B. D. Craven, and D. Ralph, eds.), University of Ballarat, 1997, pp. 79 - 95.

41. E. Zeidler, Nonlinear Functional Analysis and its Applications III, Springer-Verlag, New York, New York, 1985. 